\title{
Colloquium: Coherently controlled adiabatic passage
}

\author{
Petr Král* \\ Department of Chemistry, University of Illinois at Chicago, Chicago, Illinois 60607, USA \\ Ioannis Thanopulos ${ }^{\dagger}$ \\ Department of Chemistry, The University of British Columbia, Vancouver, \\ British Columbia, Canada V6T $1 Z 1$
}

Moshe Shapiro ${ }^{\ddagger}$

Departments of Chemistry and Physics, The University of British Columbia, Vancouver, British Columbia, Canada V6T $1 Z 1$ and Department of Chemical Physics, The Weizmann Institute of Science, Rehovot 76100, Israel

(Published 2 January 2007)

\begin{abstract}
The merging of coherent control (CC) and adiabatic passage (AP) and the type of problems that can be solved using the resulting coherently controlled adiabatic passage (CCAP) method are discussed. The discussion starts with the essence of $\mathrm{CC}$ as the guiding of a quantum system to arrive at a given final state via a number of different quantum pathways. The guiding is done by "tailor-made" external laser fields. Selectivity in a host of physical and chemical processes is shown to be achieved by controlling the interference between such quantum pathways. The AP process is then discussed, in which a system is navigated adiabatically along a single quantum pathway, resulting in a complete population transfer between two energy eigenstates. The merging of the two techniques (CCAP) is shown to achieve both selectivity and completeness. Application of CCAP to the solution of the nondegenerate quantum control problem is first discussed and shown that it is possible to completely transfer population from an initial wave packet of arbitrary shape, composed of a set of nondegenerate energy eigenstates, to a final arbitrary wave packet, also composed of nondegenerate states. The treatment is then extended to systems with degenerate states and shown how to induce isomerization between the broken-symmetry local minima of a Jahn-Teller $\mathrm{Al}_{3} \mathrm{O}$ molecule. These approaches can be further generalized to situations with many initial, intermediate, and final states and applied to quantum coding and decoding problems. CCAP is then applied to cyclic population transfer (CPT), induced by coupling three states of a chiral molecule in a cyclic fashion, $|1\rangle \leftrightarrow|2\rangle \leftrightarrow|3\rangle \leftrightarrow|1\rangle$. Interference between two adiabatic pathways in CPT allows for a complete population transfer, coupled with multichannel selectivity, by virtue of its phase sensitivity. CPT can be used to show the purification of mixtures of right-handed and left-handed chiral molecules. Finally, quantum-field coherent control is introduced, where CCAP is extended to the use of nonclassical light. This emerging field may be used to generate new types of entangled radiation-matter states.
\end{abstract}

DOI: 10.1103/RevModPhys.79.53

PACS number(s): $32.80 . \mathrm{Qk}, 33.80 .-\mathrm{b}, 42.50 . \mathrm{Hz}$

\section{CONTENTS}

I. Introduction to Quantum Control

II. Coherent Control vs Adiabatic Passage

A. Coherent control

B. Adiabatic passage

III. Nondegenerate Quantum Control Problem

IV. Quantum Control Problem in the Presence of Degenerate States

V. Coherently Controlled Quantum Adiabatic Encoding and Decoding

VI. Purification of Mixtures of Right-Handed and Left-Handed Chiral Molecules

\footnotetext{
*Electronic address: pkral@uic.edu

†Electronic address: ioannis@chem.ubc.ca

†Electronic address: moshe.shapiro@weizmann.ac.il
}

VII. Summary 70

Acknowledgments

Appendix A: Quantum-Field Coherent Control: Preparation of Entangled States

Appendix B: Derivation of the Transport Equations for

Polychromatic Passage Schemes

Appendix C: Adiabatic Evolution in Polychromatic Passage

Schemes

Appendix D: Selection Rules in Optical Transitions

73

\section{INTRODUCTION TO QUANTUM CONTROL}

The quantum control (QC) problem can be phrased as finding ways of inducing complete transfer of population from an arbitrary initial wave packet of quantum states to a desired target wave packet of quantum states. This objective can be achieved by employing a (polychro- 
matic) set of mutually coherent light fields or, equivalently, a set of tailor-made shaped laser pulses.

In recent years QC has been realized by using a number of strategies, such as coherent control (CC) (Brumer and Shapiro, 1986; Shapiro and Brumer, 2003), which achieves final-state selectivity using laser-controlled interferences between two or more quantum pathways, by tuning the phases and other parameters of the light field: optimal control (OC) (Tannor et al., 1986; Peirce et al., 1988; Kosloff et al., 1989; Judson and Rabitz, 1992; Rice and Zhao, 2000), which is a general procedure for optimizing laser pulses using pulse-shaping techniques (Weiner and Heritage, 1987; Haner and Warren, 1988; Weiner et al., 1990; Hillegas et al., 1994; Weiner, 2000) to achieve a desired outcome, and adiabatic passage (AP) (Grischkowski et al., 1975; Oreg et al., 1984; Gaubata et al., 1990), which is a way of affecting complete population transfer between selected states and, recently, also their coherent superpositions.

In parallel with the study of the control of atomic and molecular systems, quantum engineering of light states has also been extensively investigated (Peřina, 1984; Law and Eberly, 1996; Scully and Zubairy, 1997). These studies have been aided by the availability of ultracold systems and advances in solid-state electronics. In particular, numerous nonclassical light states with squeezed fluctuations of observables have been constructed and general methods for their preparation have been developed (Yurke and Stoler, 1986; Král, 1990a, 1990b; Schleich et al., 1991).

A constant flow of ideas, methods, and people between these fundamental research fields led to their explosive development. Stimulated by these achievements, the field of quantum computation also emerged, which explores possible ways to implement quantum information processing (Nielsen and Chuang, 2000). Eventually, it became clear that quantum systems could be fully controlled only if all their eigenstates (degenerate or nondegenerate, depending on the situation), such as individual material eigenstates or Fock states associated with bosonic fields, are individually addressed and populated without restrictions.

The results presented below underline the above vision in its full extent. Our emphasis in searching for useful control strategies is thus to look for general methods that can be applied to an entire class of systems. In addition to generality, the methods should ideally also be robust, i.e., insensitive to small variations of the experimental parameters, and be based on some simple and transparent principles, thus making the underlying dynamics intuitively understandable. For this reason, purely numerical approaches, such as those implicit in the optimal control field, which solve the control problem in the context of a particular system by looking for the most efficient pulse regarding a predefined objective (Rice and Zhao, 2000), are not discussed here. The merging of coherent control, by which one may attain selectivity in multichannel situations, and adiabatic passage, which enables the complete transfer of populations between a pair of quantum states, in a technique we term coherently controlled adiabatic passage (CCAP), is the central theme of this Colloquium.

In Sec. II, we briefly discuss CC and AP. In Sec. III we introduce CCAP as a way of preparing arbitrary superpositions of nondegenerate energy eigenstates. In Sec. IV, CCAP is extended to include degenerate states in either the initial or target wave packets. In Sec. V, we explain how CCAP can be used for encoding and decoding of quantum information. In Sec. VI, we show how CCAP can be used for purification of statistical mixtures of degenerate states. Conclusions are drawn in Sec. VII. In Appendix A, we extend CCAP to the nonclassical light domain. The polychromatic adiabatic passage is derived in Appendix B, and conditions for adiabatic evolution in such schemes are explained in Appendix C. General selection rules are discussed in Appendix D.

\section{COHERENT CONTROL VS ADIABATIC PASSAGE}

\section{A. Coherent control}

Coherent control (Brumer and Shapiro, 1986; Shapiro and Brumer, 2003) is a method of selecting between different (energetically allowed) outcomes using multiply interfering quantum pathways. The method is most effective in dealing with degenerate quantum states, such as those associated with systems possessing multiple continua, existing in molecular dissociation, ionization, and reactive scattering processes.

Starting from an initial pure quantum state one uses laser fields to control the multiplicity of quantum pathways which guide the population to different superpositions of the final states of interest. By inducing constructive (destructive) interference between such quantum pathways, it is possible to enhance (suppress) desired (undesired) target states. Although CC has in the past been implemented in the perturbative regime in combined fields, its validity has been shown to extend to the strong field regime as well (Shapiro and Brumer, 2003).

Control of the interferences between different pathways may be achieved experimentally by varying the relative phase between the different laser modes used to excite the system along these pathways. Although the partial coherence of light sources combined with the fast decoherence of quantum processes in material systems, due to various scattering processes, is detrimental to the control (Shapiro and Brumer, 2003), experience shows that as long as the separation of the light pulses associated with the excitation processes along different pathways is shorter than the decoherence rates, extensive control is achievable.

Many of the CC theoretical predictions, such as control of atomic and molecular processes via $N$ vs $M$ photon transitions (Shapiro et al., 1988), have been tested and demonstrated experimentally (Chen et al., 1990; Park et al., 1991; Lu et al., 1992; Yin et al., 1992; Kleiman et al., 1995; Zhu, Kleiman, Li, Lu, Trentelman, and Gordon, 1995; Wang et al., 1996; Zhu, Suto, Fiss, Wada, Seideman, and Gordon, 1997; Gordon et al., 1999). Co- 
herent control methods have also proved to be valid in the context of solid-state systems. In particular, it was shown that excitation by $N$ and $M$ multiphoton processes, having opposite parities, leads to symmetry breaking and the generation of dc electric currents (Kurizki et al., 1989; Atanasov et al., 1996; Deyirmenjian and Sipe, 1999; Král and Tománek, 1999; Král and Sipe, 2000). These predictions have been confirmed experimentally in a number of semiconductors (Baranova et al., 1990; Dupont et al., 1995; Sheehy et al., 1995; Haché et al., 1997). Similar techniques were shown to lead to the control of phonon emission (Xu and Pötz, 1999) or injection of spin currents (Bhat and Sipe, 2000).

Coherent control of photonic processes such as spontaneous emission has also been studied. Among the scenarios explored are interferences between overlapping resonances (Frishman and Shapiro, 2001, 2003) and direct interference between emission pathways (Zhu, Chan and Lee, 1995; Zhu and Scully, 1996; Zhu, Chen, and Huang, 1997; Berman, 1998; Paspalakis and Knight, 1998; Li and Zhu, 1999). The suppression of spontaneous emission in free-space (Zhu, Chan, and Lee, 1995) or band-gap materials (Zhu, Chen, and Huang, 1997) results in the spectral narrowing, even the complete elimination, of spectral lines (Zhu and Scully, 1996; Berman, 1998).

Two-photon processes can also be modified using CC. It is possible to selectively inhibit or enhance twophoton absorption by phase modulation (Yelin et al., 1997; Dayan et al., 2001, 2004) and other interference techniques (Agarwal and Harshawardhan, 1996; Gao et al., 2000). It is also possible to use CC to induce population trapping and gain in V-type systems (Paspalakis et al., 1998; Menon and Agarwal, 1999). Furthermore, it has been realized that interference between one-photon and $N$-photon transitions results in electromagnetically induced transparency (EIT) in atomic systems (Ye et al., 2002; Zibrov et al., 2002; Hu et al., 2003).

Coherent control thus provides a satisfactory solution to quests dating back to the early 1970 s of using lasers to affect selectivity. Most experiments of those days attempted to achieve selectivity by using frequency control as afforded by narrow-band lasers. Since such lasers lead to preferential excitation of atomic and molecular levels that are in resonance with the laser lines (Kurzel and Steinfeld, 1970; Bergmann and Demtröder, 1971), the hope was that such energy selectivity will also result in selectivity of subsequent events such as ionization and dissociation. This turned out to be realizable only partly due to the lack of high selectivity in addressing degenerate quantum states in overlapping continua and the existence of intramolecular vibrational redistribution (IVR) (Parmenter and Stone, 1986; Quack, 1990; Crim, 1993; Moss and Parmenter, 1993; Borst and Pratt, 2000), a process by which the narrow wave packet of states excited by the narrow-band laser pulses quickly disperses to many molecular vibrational modes.

In order to understand better the concepts developed below, we briefly discuss here a sample CC scenario in which we interfere a one-photon process with a two-

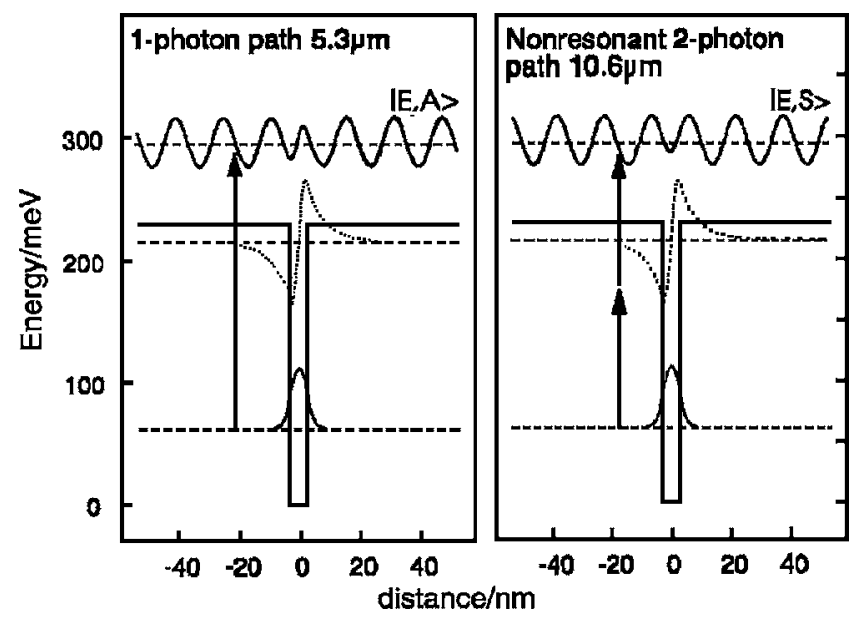

FIG. 1. A schematic illustration of an experiment in which the directionality of current formed in the conduction band of a semiconducting quantum well system was controlled by irradiating the sample by a combination of a fundamental frequency (that of the $\mathrm{CO}_{2}$ laser) and its second harmonics. From Dupont et al., 1995.

photon process in order to photoinject electric currents in semiconductors or other environments and control their directionality (Kurizki et al., 1989). The experimental realizations of this scenario [see, e.g., Dupont et al. (1995)], shown in Fig. 1, proceed along the following theoretical scenario: One simultaneously irradiates the material system by two electromagnetic fields, which in the case of semiconductors is convenient to describe by vector potentials using the $\mathbf{p} \cdot \mathbf{A}$ gauge of the radiationmatter interaction Hamiltonian (Shore, 1990; Scully and Zubairy, 1997). One potential $A_{1} e^{-i \omega_{1} t-i \phi_{1}}$ is inducing one-photon transitions, and the other $A_{2} e^{-i \omega_{2} t-i \phi_{2}}$, where $\omega_{2}=\omega_{1} / 2$, is inducing two-photon transitions. In the case from Fig. 1, the initial states are confined to a quantum well in one direction and are of even symmetry, and they are delocalized in the other two directions. The excited conduction states are delocalized in all three directions, but their components in the localization direction of the initial states have odd symmetry for one-photon processes and even symmetry for two-photon processes. These two components interfere when projected on the momentum-state amplitudes in the direction of the initial-state confinement and asymmetrically populate these states. The resulting light-induced brokensymmetry electron distributions in momentum space lead to the generation of nonzero photocurrents.

Let us now explain the phenomenon in more detail in bulk semiconductors (Král and Sipe, 2000). Given the initial $\left|\mathbf{k}_{v}\right\rangle$ valence-band states and the fact that the two interfering processes are in resonance with transitions to energetically degenerate $\left|\mathbf{k}_{c}\right\rangle$ conduction-band states (in vertical transitions, $\mathbf{k} \equiv \mathbf{k}_{c} \approx \mathbf{k}_{v}$ ), the probability density in the conduction band $P(\mathbf{k})=|f(\mathbf{k})|^{2}$ is given as the square of the sum of one-photon and two-photon amplitudes.

Using the rotating-wave approximation (RWA) (Shore, 1990; Scully and Zubairy, 1997) [see also discus- 
sion below Eq. (9)] and the lowest-order perturbation theory in the combined fields ${ }^{1}$ (Král and Sipe, 2000), these amplitudes are given by

$$
f(\mathbf{k}) \propto A_{1} p_{c v}(\mathbf{k}) e^{-i \phi_{1}}+A_{2}^{2} d_{c v}(\mathbf{k}) e^{-2 i \phi_{2}},
$$

where

$$
p_{c v}(\mathbf{k}) \equiv\left\langle\mathbf{k}_{c}|p| \mathbf{k}_{v}\right\rangle, \quad d_{c v}(\mathbf{k}) \equiv \sum_{i} \frac{p_{c i}(\mathbf{k}) p_{i v}(\mathbf{k})}{\hbar \omega_{2}-E_{i}},
$$

and $p_{i v}(\mathbf{k}) \equiv\left\langle\mathbf{k}_{i}|p| \mathbf{k}_{v}\right\rangle\left[p_{c i}(\mathbf{k}) \equiv\left\langle\mathbf{k}_{c}|p| \mathbf{k}_{i}\right\rangle\right]$ are the momentum matrix elements between the initial (final) state and all intermediate states $\left|\mathbf{k}_{i}\right\rangle$ of energy $E_{i}$.

Squaring Eq. (1) and separating out the noninterfering sum of one-photon and two-photon probabilities $P_{\text {non }}(\mathbf{k}) \equiv\left|A_{1} p_{c v}(\mathbf{k})\right|^{2}+\left|A_{2}^{2} d_{c v}(\mathbf{k})\right|^{2}$ from the interference term $2 \mathcal{R}_{e}\left\{A_{1}^{*} p_{c v}^{*}(\mathbf{k}) A_{2}^{2} d_{c v}(\mathbf{k}) e^{i\left(\phi_{1}-2 \phi_{2}\right)}\right\}$, we can write $P(\mathbf{k})$ as

$$
P(\mathbf{k}) \propto P_{\text {non }}(\mathbf{k})+P_{\text {int }}(\mathbf{k}) \cos \left[\delta_{1,2}(\mathbf{k})+\phi_{1}-2 \phi_{2}\right],
$$

where $\quad P_{\text {int }}(\mathbf{k}) \equiv\left|A_{1}^{*} p_{c, v}^{*}(\mathbf{k}) A_{2}^{2} d_{c, v}(\mathbf{k})\right|, \quad$ with $\quad \delta_{1,2}(\mathbf{k})$ $=\delta_{1,2}(-\mathbf{k})+\pi$ denoting the $\mathbf{k}$-dependent phase of the $A_{1}^{*} p_{c v}^{*}(\mathbf{k}) A_{2}^{2} d_{c v}(\mathbf{k})$ product. By varying $\phi_{1}-2 \phi_{2}$ we can control the sign of the interference term and hence whether the interference is constructive (destructive) for a given excited momentum state $\left|\mathbf{k}_{c}\right\rangle$, i.e., whether it is constructive (destructive) in the forward (backward) direction, as determined by $\mathbf{k}(-\mathbf{k})$, or vice versa. The mutual shift of phases $\delta_{1,2}(\mathbf{k})$ and $\delta_{1,2}(-\mathbf{k})$ by $\pi$ (Král and Sipe, 2000) assures that when a scenario occurs for one momentum direction, the opposite scenario occurs for the opposite direction.

The injection rates into the $\left| \pm \mathbf{k}_{c}\right\rangle$ momentum states of conduction electrons leaving the excitation spot depend on the $\phi_{1}-2 \phi_{2}$ relative phase, which results in symmetry breaking of the population distribution [see Král and Sipe (2000)]. This asymmetry leads to the generation of dc electric current $\mathbf{j}_{c} \propto \int \mathbf{k}_{c} P_{\mathbf{k}_{c}} d \mathbf{k}_{c} \neq 0\left(\mathbf{j}_{v} \neq \mathbf{j}_{c}\right)$ in the conduction (valence) band of the semiconductor. In Fig. 2, we also show the $\phi_{1}-2 \phi_{2}$ phase dependence of the experimentally measured current (Dupont et al., 1995) induced in this way. Its direction can be clearly controlled by the relative phase $\phi_{1}-2 \phi_{2}$.

\section{B. Adiabatic passage}

With the introduction of new laser sources in the 1980s a number of powerful population transfer techniques were developed, such as overtone pumping (Crim, 1984), off-resonance stimulated Raman scattering (Orr et al., 1984; Meier et al., 1986), and stimulated emission pumping (Kittrell et al., 1981). These methods resulted, however, in only partial population transfer between quantum states. The adoption of the adiabatic

\footnotetext{
${ }^{1}$ The one-photon excitation due to the $\mathbf{A}_{2}$ vector potential is nonresonant due to the band gap, and the two-photon excitation due to the $\mathbf{A}_{2}$ potential is of a similar strength like the one-photon excitation due to the $\mathbf{A}_{1}$ potential.
}

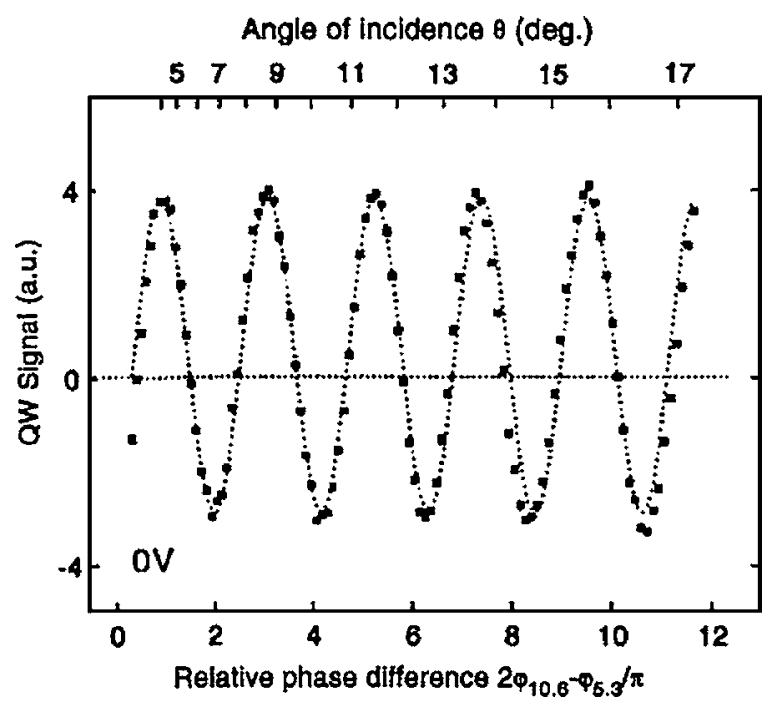

FIG. 2. Experimental control of current directionality. Shown is the dc current generated by the experimental setup of Fig. 1 as a function of the phase difference between the fundamental source (wavelength of $10.6 \mu \mathrm{m}$ ) and its second harmonic. From Dupont et al., 1995.

passage technique (Oreg et al., 1984; Gaubatz et al., 1988, 1990), operating (in contrast to CC) along a single quantum pathway, has opened the way for inducing a complete population transfer between quantum material states.

Complete population transfer in AP was shown theoretically (Oreg et al., 1984) and independently implemented experimentally a few years later in a method called stimulated Raman adiabatic passage (STIRAP) (Gaubatz et al., 1988, 1990). In this method, which is a variant of the stimulated Raman process, two (pump and Stokes) pulses are employed in the reverse ("counterintuitive") order to that of the usual stimulated Raman process; namely, in STIRAP the Stokes pulse precedes the pump pulse. This method, designed to work with discrete states, clearly complements $\mathrm{CC}$ by being able to transfer populations completely but at the same time not being phase sensitive, in contrast to the $\mathrm{CC}$ that is typically used in continua of states in weak-field but phasesensitive regimes. We will later explore the advantages resulting from the combination of both approaches.

We now show that the counterintuitive pulse ordering leads to the desired complete population transfer. Consider irradiating the ( $\Lambda$-type) three-state system depicted in Fig. 3 (left) by two laser pulses, a pump pulse $(P)$ of center frequency $\omega_{P}$ and electric field envelope $\mathcal{E}_{P}(t)$ and a Stokes pulse $(S)$ of center frequency $\omega_{S}$ and field envelope $\mathcal{E}_{S}(t)$. We assume that the pump pulse is close to resonance with the $|1\rangle \leftrightarrow|0\rangle$ transition and the Stokes pulse is close to resonance with the $|2\rangle \leftrightarrow|0\rangle$ transition, and the two transitions have well separated energies.

The time-dependent Hamiltonian of this radiationmatter system can be written in the Schrödinger picture as follows (Shore, 1990; Scully and Zubairy, 1997): 
10)

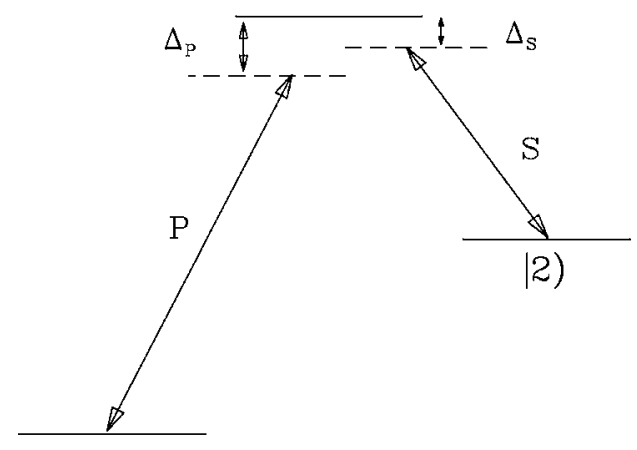

1)
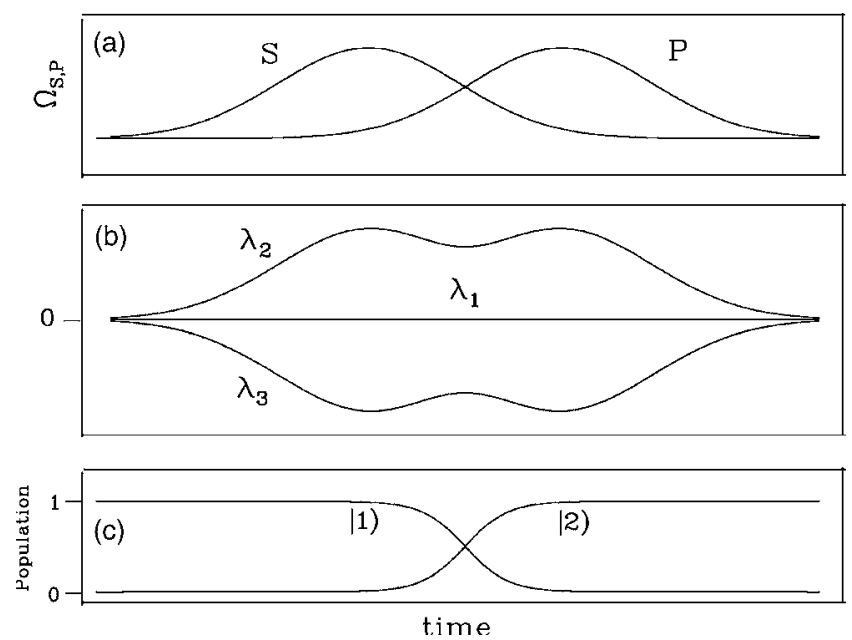

FIG. 3. The model and dynamics in the adiabatic passage. (Left) The three-level $\Lambda$ configuration adiabatic passage scheme. (Right) The pulses [panel (a), where $P$ stands for the pump and $S$ for the Stokes pulse], eigenvalues [panel (b)], and populations [panel(c)] as a function of time in this adiabatic passage with counterintuitive pulse ordering.

$$
\begin{aligned}
H(t)= & H_{M}+H_{I}(t), \quad H_{M}=\sum_{i=1,0,2} E_{i}|i\rangle\langle i|, \\
H_{I}(t)= & -\mathcal{E}_{P}(t) \cos \left(\omega_{P} t\right)\left(\mu_{0,1}|0\rangle\langle 1|+\text { H.c. }\right) \\
& -\mathcal{E}_{S}(t) \cos \left(\omega_{S} t\right)\left(\mu_{0,2}|0\rangle\langle 2|+\text { H.c. }\right) .
\end{aligned}
$$

Here, as well as in all subsequent sections, we use the $\mathbf{r} \cdot \mathbf{E}$ gauge of the radiation-matter interaction Hamiltonian $H_{I}(t)$. In Eq. (4), $\mu_{0,1}$ is the projection of the dipole matrix element for the $|1\rangle \leftrightarrow|0\rangle$ transition on the $P$ polarization axis, $\mu_{2,0}$ is the projection of the dipole matrix element for the $|0\rangle \leftrightarrow|2\rangle$ transition on the $S$ polarization axis, and $E_{i}=\hbar \omega_{i}$ are the free-field eigenenergies. The connection between the electric field envelope $\mathcal{E}(t)$ and the intensity of the radiation $I(t)$ is

$$
\mathcal{E}(t)[\mathrm{V} / \mathrm{cm}]=27.4682 \sqrt{I(t)\left[\mathrm{W} / \mathrm{cm}^{2}\right]} .
$$

We can write the time-dependent wave function $|\Psi(t)\rangle$ solving the Schrödinger equation

$$
\hbar \frac{d}{d t}|\Psi(t)\rangle=-i H(t)|\Psi(t)\rangle
$$

in the form (Shore, 1990; Scully and Zubairy, 1997)

$$
|\Psi(t)\rangle=\sum_{i=1,0,2} C_{i}(t)|i\rangle=\sum_{i=1,0,2} c_{i}(t) e^{-i \omega_{i} t}|i\rangle .
$$

After substituting this $|\Psi(t)\rangle$ into Eq. (6) and performing its time derivative on the left side, we can cancel the terms associated with the free evolution in the $H_{M}$ Hamiltonian. Then, its multiplication by the terms $\langle j| e^{i \omega_{j} t}$ gives a set of equations for the $c_{i}(t)$ coefficients. If we collect these coefficients in the vector $\mathbf{c}(t)$ $\equiv\left(c_{1}(t), c_{0}(t), c_{2}(t)\right)^{\mathrm{T}}$, where the superscript $\mathrm{T}$ designates the matrix transpose, Eq. (6) can be written in the matrix form

$$
\hbar \frac{d}{d t} \mathbf{c}(t)=-i \mathrm{H}(t) \cdot \mathbf{c}(t),
$$

where

$$
\mathrm{H}(t)=\frac{\hbar}{2}\left(\begin{array}{ccc}
0 & \Omega_{P}^{*}(t) e^{-i \Delta_{P} t} & 0 \\
\Omega_{P}(t) e^{i \Delta_{P} t} & 0 & \Omega_{S}^{*}(t) e^{i \Delta_{S} t} \\
0 & \Omega_{S}(t) e^{-i \Delta_{S} t} & 0
\end{array}\right) .
$$

This is the Schrödinger equation in the interaction representation for the problem described by the Hamiltonian in Eq. (4) (Shore, 1990; Scully and Zubairy, 1997). We present its formal derivation in Appendix B.

In Eq. (9), $\Omega_{\nu} \equiv \mu_{\alpha} \mathcal{E}_{\nu} / \hbar[\nu=P, S ; \alpha=(0,1),(2,0)]$ is the Rabi frequency for the pump (Stokes) pulse and $\Delta_{\nu}$ $\equiv \omega_{\nu}-\omega_{\alpha}(\nu=P, S)$ its detuning relative to the transition frequency $\omega_{\alpha}=\omega_{0}-\omega_{\beta}[\alpha=(0,1),(2,0), \beta=1,2]$ between levels $|0\rangle$ and $|1\rangle(|0\rangle$ and $|2\rangle)$. Even though the Rabi frequencies are in general complex, in some of the cases discussed below we choose them, for simplicity, to be real.

In deriving the Hamiltonian $\mathrm{H}(t)$, we have also employed the RWA, according to which one only keeps terms proportional to the slowly varying factors $\exp \left[ \pm i t\left(\omega_{\nu}-\left(E_{i}-E_{j}\right) / \hbar\right]\right.$, where $\omega_{\nu} \approx\left(E_{i}-E_{j}\right) / \hbar(\nu=P, S)$, while we neglect terms containing the rapidly oscillating factors $\exp \left\{ \pm i t\left[\omega_{\nu}+\left(E_{i}-E_{j}\right) / \hbar\right]\right\} \quad(\nu=P, S)$. Since in all cases studied here the excitation fields are in resonance $\left(\Delta_{P}=\Delta_{S}=0\right)$ with all considered states, the RWA Hamiltonian $\mathrm{H}(t)$ does not possess oscillatory terms, and we can use the concept of adiabatic states (Shore, 1990; Scully and Zubairy, 1997).

We will diagonalize now the RWA Hamiltonian $\mathrm{H}(t) \cdot \mathbf{U}(t)=\mathbf{U}(t) \cdot \hat{\lambda}(t)$, where $\hat{\lambda}(t)$ is a diagonal matrix with coefficients

$$
\lambda_{1}=0, \lambda_{2,3}(t)= \pm\left[\left|\Omega_{P}(t)\right|^{2}+\left|\Omega_{S}(t)\right|^{2}\right]^{1 / 2} .
$$

The normalized eigenvector corresponding to the $\lambda_{1}=0$ null eigenvalue is 


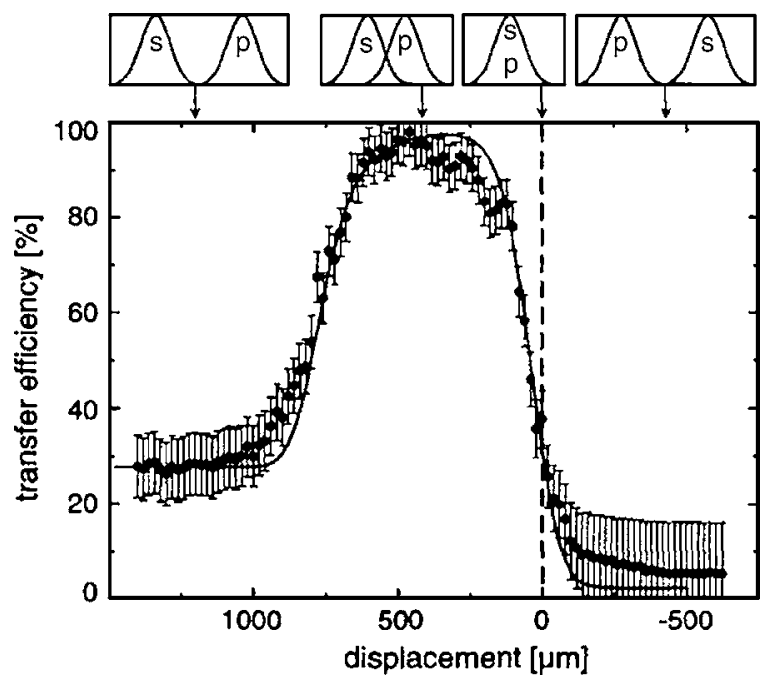

FIG. 4. Experiments on STIRAP in the Ne atom. Shown is the percentage of population transfer to level $|2\rangle\left({ }^{3} P_{2}\right)$ as a function of the delay between the pump and Stokes pulses. As the pulses begin to overlap, with the Stokes pulse preceding the pump pulse, the population transfer to the $|2\rangle$ state approaches 100\%. From Bergmann et al., 1998.

$$
\mathrm{U}^{(1)}(t)=\left(\begin{array}{c}
\cos \theta(t) \\
0 \\
-e^{i \chi(t)} \sin \theta(t)
\end{array}\right), \quad \theta(t)=\arctan \left(\left|\frac{\Omega_{P}(t)}{\Omega_{S}(t)}\right|\right)
$$

where $\chi(t) \equiv \phi_{P}(t)-\phi_{S}(t)$ and $\phi_{i}(t)$ are the phases of the Rabi frequencies, $\Omega_{i}(t) \equiv\left|\Omega_{i}(t)\right| e^{i \phi_{i}(t)} \quad(i=P, S)$, allowing the formation of null eigenvalue (dark) states described below.

We can write the dark state in the interaction representation and the bare-state basis $|i\rangle(i=1,0,2)$ as

$$
\left|\lambda_{1}(t)\right\rangle=\cos \theta(t)|1\rangle-\sin \theta(t) e^{i \chi(t)}|2\rangle
$$

and transform it to the Schrödinger picture as follows:

$$
\begin{aligned}
\left|\Lambda_{1}(t)\right\rangle & =U_{0}(t)\left|\lambda_{1}(t)\right\rangle \\
& =\cos \theta(t) e^{-i \omega_{1} t}|1\rangle-\sin \theta(t) e^{-i \omega_{2} t+i \chi(t)}|2\rangle,
\end{aligned}
$$

where $U_{0}(t)=\exp \left(-i H_{M} t / \hbar\right)$ (see Appendix B). Since the other two dark states $\left|\lambda_{\nu}(t)\right\rangle(\nu=2,3)$ turn out not to be connected at $t=0$ to the $|1\rangle$ state, we see from Eqs. (11) and (12) that a system residing initially in state $|1\rangle$ correlates exclusively with the $\left|\lambda_{1}(t)\right\rangle$ dark eigenvector. Moreover, if the Stokes pulse is made to precede the pump pulse, this eigenvector executes a smooth transition from state $|1\rangle$ to $|2\rangle$, while leaving state $|0\rangle$ unpopulated throughout the process. Thus, as illustrated in Fig. 3 (right), we can achieve the desired complete population transfer.

In the continuous regime, where flying molecules cross mutually space shifted light beams, STIRAP has been demonstrated for $\mathrm{Na}_{2}$ (Gaubatz et al., 1988, 1990) and, as shown in Fig. 4, for $\mathrm{Ne}^{*}$ atoms (Theuer and Bergmann, 1998) and, in the pulsed regime, for NO (Kuhn et al., 1998) and $\mathrm{SO}_{2}$ (Halfmann and Bergmann, 1996). It has been demonstrated in the "ladder" configuration for $\mathrm{Rb}$ (Süptitz et al., 1997) and with degenerate or nearly degenerate states (Martin et al., 1996).

Following the initial works of Bergmann and others, the properties of three-state STIRAP have been thoroughly investigated theoretically (Oreg et al., 1984; Kuklinski et al., 1989; Shore, 1995; Vitanov et al., 2001). Among the properties examined were the sensitivity of the population transfer to the delay between the Stokes and pump pulses (Bergmann et al., 1998) and the effect of single-photon (Vitanov and Stenholm, 1997a) and two-photon (Vitanov et al., 2001) detuning. Going beyond the RWA (Guérin and Jauslin, 1998; Yatsenko et al., 1998), parasitic effects due to losses from intermediate states (Band and Julienne, 1991, 1992; Glushko and Kryzhanovsky, 1992; Fleischhauer and Manka, 1996; Vitanov and Stenholm, 1997b) and the existence of many intermediate states (Coulston and Bergmann, 1992; Vitanov and Stenholm, 1999) have also been studied.

Generalizations of the three-state STIRAP to multistate chains (Marte et al., 1991; Shore et al., 1991; Malinovsky and Tannor, 1997; Law and Eberly, 1998; Theuer and Bergmann, 1998; Nakajima, 1999), to adiabatic momentum transfer (Marte et al., 1991; Esslinger et al., 1996; Kulin et al., 1997; Theuer and Bergmann, 1998), to branched-chain excitation (Kobrak and Rice, 1998a, 1998b, 1998c; Unanyan, Vitanov, and Stenholm, 1998; Theuer et al., 1999), and to population transfer via a continuum of intermediate states (Carroll and Hioe, 1995, 1996; Paspalakis et al., 1997; Vitanov and Stenholm, 1997c; Yatsenko et al., 1997; Unanyan, Vitanov, and Stenholm, 1998; Unanyan et al., 2000), related to laser-induced continuum structure (LICS) (Knight et al., 1990; Halfman et al., 1998; Kylstra et al., 1998; Yatsenko, Halfmann, Shore, and Bergmann, 1999) have also been made.

Numerous novel techniques related to STIRAP have been further developed recently. An example is the hyper-Raman STIRAP (Guérin and Jauslin, 1998; Guérin et al., 1998; Yatsenko et al., 1998), Stark-chirped rapid adiabatic passage (Yatsenko, Shore, Halfmann, Bergmann, and Vardi, 1999; Rickes et al., 2001), adiabatic passage by light-induced potentials (Garraway and Suominen, 1998; Kallush and Band, 2000; Rodriguez et al., 2000; Solá et al., 2000), and photoassociative STIRAP, as a source for cold molecules (Vardi et al., 1997, 1999; Javanainen and Mackie, 1998; Mackie et al., 2000). Some experimental implementations of these ideas, e.g., to the formation of dark states in the photoassociation of an atomic Bose-Einstein condensate (BEC) to form a molecular BEC, have already been reported (Dumke et al., 2005; Winkler et al., 2005).

\section{NONDEGENERATE QUANTUM CONTROL PROBLEM}

So far, we have considered separately the topics of selectivity control, i.e., the introduction of an active bias which selects out of a multiplicity of possible events one desired outcome, and the attainment of a complete population transfer from one energy eigenstate to an- 
other. In the former, as practiced in the perturbative version of CC, the transfer of population between the initial and final states is far from complete. In the latter, as attained in three-state STIRAP, the possibility of a number of final outcomes is usually not considered.

In this section, we combine these two approaches in a method called nondegenerate quantum control (NQC), where the goal is to achieve complete population transfer between two arbitrary superposition states (wave packets) composed of many nondegenerate energy eigenstates. The present emphasis on the nondegenerate nature of constituent states stems from the fact that in this case frequency discrimination allows for an easy and simple addressing of each constituent state. Such a simple means of addressing is not possible if degeneracies exist, and we postpone the discussion of the more complicated case of quantum control between superpositions of degenerate energy eigenstates to Sec. IV.

In spite of the above limitations, nondegenerate quantum control is of interest in many atomic and molecular systems [see, e.g., Beumee and Rabitz (1990), Aubanel and Bandrauk (1997), de Araujo (1999)]. Although a theorem has been published (Huang et al., 1983; Rice and Zhao, 2000), asserting that a solution to the NQC problem exists under certain conditions, this existence theorem provides no clue as to the nature of the external field which actually brings about this type of population transfer.

Solutions of the NQC problem for some particular weak-field CC cases (Shapiro and Brumer, 1999, 2003), the three-state STIRAP (Grischkowski et al., 1975; Gaubatz et al., 1990; Kuhn et al., 1992; Bergman et al., 1998; Theuer et al., 1999; Vitanov et al., 2001), and its generalization to a few more final states (Coulston and Bergman, 1992; Kobrak and Rice, 1998a, 1998b, 1998c; Unanyan, Fleischhauer, Shore, and Bergmann, 1998; Král et al., 2001) have been known for quite some time. The construction of fields which solve the NQC problem has been achieved recently (Král et al., 2002). Although this construction is not the only one possible, its existence is enough to establish that the NQC problem is practically solvable. As it turns out, this construction is simple and analytic, making it especially attractive and useful for laboratory implementations.

The solution given by Král et al. (2002) is a generalization of the three-state STIRAP (Grischkowski et al., 1975; Gaubatz et al., 1990; Kuhn et al., 1992; Bergmann et al., 1998; Theuer et al., 1999; Vitanov et al., 2001), discussed in Sec. II.B above. It has evolved from the intermediate result (Král and Shapiro, 2002), dealing with the transfer of population from a single energy eigenstate $|1\rangle$ to a wave packet composed of nondegenerate energy eigenstates with arbitrary coefficients $\left|\Psi^{\prime}(t)\right\rangle=\Sigma_{k} c_{k} e^{-i \omega_{k} t}|k\rangle(k \neq 1)$. As in three-state STIRAP, the population transfer proceeds via an intermediateenergy eigenstate $|0\rangle$, which acts as a virtual unpopulated "stepping stone." The method as applied to the preparation of vibrational coherent wave packets on the ground electronic state of the $\mathrm{Na}_{2}$ molecule (Král and Shapiro, 2002) is outlined in Appendixes B-D. In an

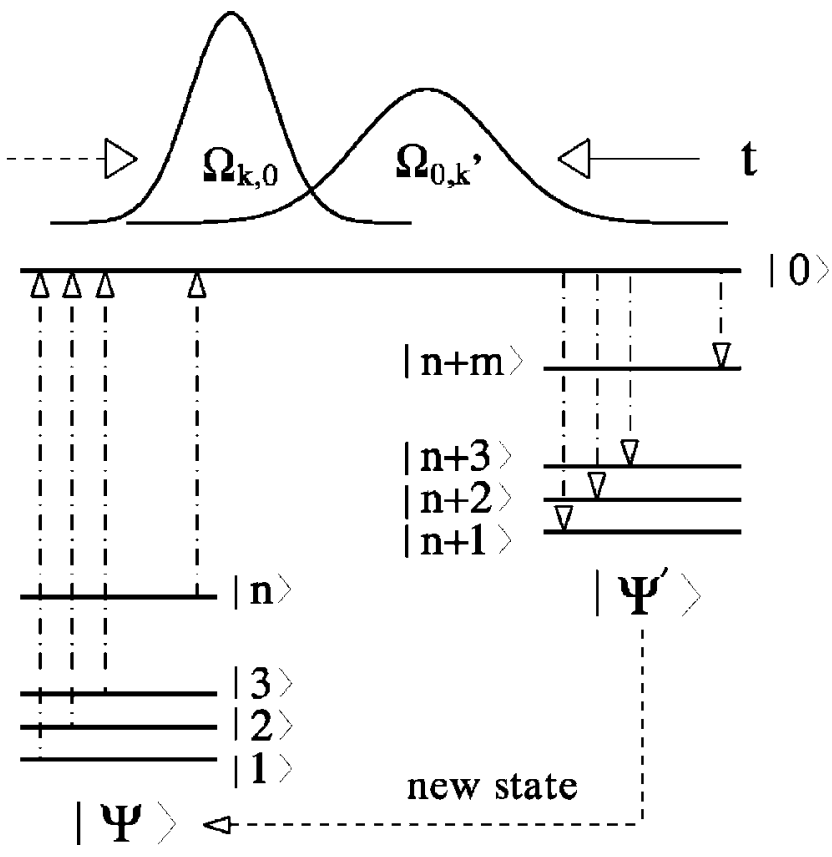

FIG. 5. An illustration of a transfer process in which arbitrary wave packets made of nondegenerate states can be populated over and over, $|\Psi\rangle \rightarrow|0\rangle \rightarrow\left|\Psi^{\prime}\right\rangle$.

analogous way, one could prepare arbitrary nonclassical vibrational states by controlling all vibrational (Fock) eigenstates individually (Král, 1990a).

The solution of the full NQC problem, i.e., the achievement of the $\left|\Psi^{i}\left(t_{\text {in }}\right)\right\rangle=\Sigma_{k} c_{k}^{i}\left(t_{\text {in }}\right) e^{-i \omega_{k} t_{\text {in }}}|k\rangle$ $\rightarrow\left|\Psi^{f}\left(t_{\text {end }}\right)\right\rangle=\Sigma_{l} c_{l}^{f}\left(t_{\text {end }}\right) e^{-i \omega_{l} t_{\text {end }} \mid}|l\rangle$ transfer process, can be accomplished in a straightforward manner (Král et al., 2002), where it is also possible to control the extent of population transfer, from 0 to $100 \%$. [Control over the extent of transfer can also be achieved in other ways, e.g., by successive transitions using a chain of states (Malinovsky and Tannor, 1997).]

Figure 5 illustrates the present method, which consists of applying two pulses in a counterintuitive order. The Stokes pulse, characterized by a set of Rabi frequencies $\Omega_{0, l}$, coupling the intermediate $|0\rangle$ state to the final $|l\rangle$ $(l=n+1, \ldots, n+m)$ states, is made to precede (while partially overlapping in time) the pump pulse, whose constituent Rabi frequencies $\Omega_{k, 0}$ couple the initially populated $|k\rangle(k=1, \ldots, n)$ states and the intermediate $|0\rangle$ state.

We therefore consider the action of two pulses, whose electric field is given as

$$
\vec{E}(t)=\operatorname{Re} \sum_{k=1}^{n+m} \hat{\epsilon}_{k} \mathcal{E}_{k}(t) e^{-i \omega_{0, k} t}
$$

on a wave packet composed of $|1\rangle, \ldots,|n\rangle$ states with $\hbar \omega_{1}, \ldots, \hbar \omega_{n}$ eigenenergies. In Eq. (14), $\mathcal{E}_{k}(t)$ are the (slowly varying) envelopes of the electromagnetic field modes $\omega_{0, k}$, each polarized along the $\hat{\epsilon}_{k}$ direction. For simplicity, we will use for the field mode frequencies the same symbols as for the transition frequencies $\omega_{0, k}=\omega_{0}$ 
$-\omega_{k}$, introduced in Eq. (9), since the former are tuned to be in resonance with the latter.

The first $n$ components of $\vec{E}(t)$ describe the pump pulse and the last $m$ components the Stokes pulse. The time dependence of the common Stokes (pump) pulse envelope is controlled by the function $0<f_{\nu}(t)<1$ $(\nu=S, P)$ according to $\mathcal{E}_{l}(t)=f_{S}(t) \eta_{l}$ for $l=n+1, \ldots, n+m$ and $\mathcal{E}_{k}(t)=f_{P}(t) \eta_{k}$ for $k=1, \ldots, n$, with $\eta_{i}=\max \left\{\mathcal{E}_{i}(t)\right\}$ $(i=1, \ldots, n+m)$.

The time-dependent radiation-matter Hamiltonian assumes the form

$$
\begin{aligned}
& H(t)=H_{M}+\frac{\hbar}{2} \operatorname{Re} \sum_{k=1}^{n+m}\left[\Omega_{0, k}(t) e^{-i \omega_{0, k} t}|0\rangle\langle k|+\text { H.c. }\right], \\
& \Omega_{0, k}(t) \equiv \mu_{0, k} \mathcal{E}_{k}(t) / \hbar=\mu_{0, k} \eta_{k} f_{\nu}(t) / \hbar \equiv \mathcal{O}_{0, k} f_{\nu}(t),
\end{aligned}
$$

where $\Omega_{0, k}(t) \quad(\nu=S, P)$ are the Rabi frequencies, with $\mu_{0, k}$ being the electric-dipole matrix elements between the $|0\rangle$ and $|k\rangle$ states in the polarization $\hat{\epsilon}_{k}$.

We denote by $\mathbf{c}(t) \equiv\left(c_{0}, c_{1}, \ldots, c_{n}, c_{n+1}, \ldots, c_{n+m}\right)^{\mathrm{T}}$ the vector of the time-dependent expansion coefficients of $|\Psi(t)\rangle$ in the bare states. Then, invoking the RWA and neglecting all off-resonance terms, we can derive from Eq. (15) the Schrödinger equation (8) subject to the RWA Hamiltonian, with time-dependent Rabi frequencies $\Omega_{i, j}(t)$, of the form

$$
\mathrm{H}(t)=\frac{\hbar}{2}\left[\begin{array}{ccccccc}
0 & \Omega_{0,1} & \cdots & \Omega_{0, n} & \Omega_{0, n+1} & \cdots & \Omega_{0, n+m} \\
\Omega_{1,0} & 0 & \cdots & 0 & 0 & \cdots & 0 \\
\cdots & \cdots & \cdots & \cdots & \cdots & \cdots & \cdots \\
\Omega_{n, 0} & 0 & \cdots & 0 & 0 & \cdots & 0 \\
\Omega_{n+1,0} & 0 & \cdots & 0 & 0 & \cdots & 0 \\
\cdots & \cdots & \cdots & \cdots & \cdots & \cdots & \cdots \\
\Omega_{n+m, 0} & 0 & \cdots & 0 & 0 & \cdots & 0
\end{array}\right] .
$$

This is an extension of the Hamiltonian (B5) to the case of many initial states, where we can neglect the offresonance terms as in Appendix B, due to sufficiently large level spacing $\omega_{k}-\omega_{j}(k \neq j, k, j=1, \ldots, n+m)$, compared to the Rabi frequencies used (Shore, 1990). There are no detuning parameters since the fields are assumed to be in resonance with the individual transition frequencies.

Of the $n+m+1$ eigenvalues $\lambda_{i}(t)$ of the RWA Hamiltonian $\mathrm{H}(t), n+m-1$ are zero, $\lambda_{1}=\lambda_{2}=\cdots=\lambda_{n+m-1}$ $=0$, and two are nonzero, $\lambda_{n+m}(t)=-\lambda_{n+m+1}(t)$ $=\left[\sum_{k=1}^{n+m}\left|\Omega_{0, k}(t)\right|^{2}\right]^{1 / 2}$. The crucial zero eigenvalues correspond to three types of null adiabatic eigenvalue states, which we term the initial null-eigenvector states (INS's), the mixed null-eigenvector states (MNS's), and the final null-eigenvector states (FNS's). The three types of dark states can be written in the interaction representation as in Eq. (12),

$$
\begin{aligned}
& \left|D_{k k^{\prime}}^{I}(t)\right\rangle=\Omega_{0, k^{\prime}}(t)|k\rangle-\Omega_{0, k}(t)\left|k^{\prime}\right\rangle, \\
& \left|D_{k l}^{M}(t)\right\rangle=\Omega_{0, l}(t)|k\rangle-\Omega_{0, k}(t)|l\rangle, \\
& \left|D_{l l^{\prime}}^{F}(t)\right\rangle=\Omega_{0, l^{\prime}}(t)|l\rangle-\Omega_{0, l^{\prime}}(t)\left|l^{\prime}\right\rangle \\
& \left(k \neq k^{\prime}=1, \ldots, n ; \quad l \neq l^{\prime}=n+1, \ldots, n+m\right) .
\end{aligned}
$$

These states are not yet normalized, but this does not affect their composition. Note that the phases of the time-dependent Rabi frequencies of Eq. (15), including the phases of electric fields $\mathcal{E}_{k}(t)$ and dipole elements $\mu_{0, i}$, which in the case of the single dark state were explicitly shown in $\chi(t)$ of Eq. (12), are incorporated in the time-dependent Rabi frequencies $\Omega_{0, i}(t)$ in Eq. (17). We can explicitly check that the vectors formed by the nonzero time-dependent coefficients of these states, $\mathbf{c}(t)$ $=\left(0, \ldots, \Omega_{0, i}(t), \ldots,-\Omega_{0, j}(t), \ldots\right)^{\mathrm{T}}$, are dark eigenstates of the RWA Hamiltonian (16). The construction in Eq. (17) generates $n(n-1) / 2$ INS's, $n m$ MNS's, and $m(m-1) / 2$ FNS's, out of which only $n+m-1$ states are linearly independent.

The essence of the present NQC solution is to transfer population from any wave packet made of initial states to any other wave packet made of final states. If we want to have a $100 \%$ population transfer between two such chosen wave packets, then the fields that realize this transfer are almost unambiguously defined. We can approach the problem in two different ways: either we find the fields that transfer the chosen wave packets or we assign to the given fields the wave packets that can be transferred by them.

We now discuss the second option and assign the wave packets to the fields of Eq. (14) and Hamiltonian of Eq. (16). During the transfer, the MNS's are combined in such a way that they form a single transferring dark state, which correlates (in the counterintuitive pulse ordering) at $t=t_{\text {in }}$ with the initial state $\left|\Psi^{i}\left(t_{\text {in }}\right)\right\rangle$ $=\Sigma_{k} c_{k}^{i}\left(t_{\text {in }}\right) e^{-i \omega_{k} t_{\text {in }}}|k\rangle$, and at $t=t_{\text {end }}$ with some final state $\left|\Psi^{f}\left(t_{\text {end }}\right)\right\rangle=\Sigma_{l} c_{l}^{f}\left(t_{\text {end }}\right) e^{-i \omega \omega_{l} t_{\text {end }}}|l\rangle$.

The particular combination of MNS's in the interaction representation that satisfies these asymptotic conditions is

$$
\begin{aligned}
\left|D^{M}(t)\right\rangle & =\sum_{k, l} t_{k l}\left|D_{k l}^{M}(t)\right\rangle \\
& =\sum_{k=1}^{n}|k\rangle \sum_{l=n+1}^{n+m} t_{k l} \Omega_{0, l}(t)-\sum_{l=n+1}^{n+m}|l\rangle \sum_{k=1}^{n} t_{k l} \Omega_{0, k}(t),
\end{aligned}
$$

where the $t_{k l}$ coefficients are chosen such that $\sum_{l=n+1}^{n+m} t_{k l} \Omega_{0, l}(t) \propto c_{k}^{i}(t) \quad$ and $\quad \sum_{k=1}^{n} t_{k l} \Omega_{0, k}(t) \propto c_{l}^{f}(t)$. These equations can be satisfied by choosing $\left[\Omega_{k, 0}(t)\right.$ $\left.\equiv \mathcal{O}_{k, 0} f_{\nu}(t), \nu=S, P\right]$

$$
t_{k l}=\mathcal{O}_{k, 0} \mathcal{O}_{l, 0}, \quad \Omega_{k, 0}(t) \propto \mathcal{C} c_{k}^{i}(t), \Omega_{l, 0}(t) \propto \mathcal{C}^{\prime} c_{l}^{f}(t),
$$

where $\mathcal{C}, \mathcal{C}^{\prime}$ are arbitrary complex numbers. These numbers must be small enough so that the (slowly varying) 


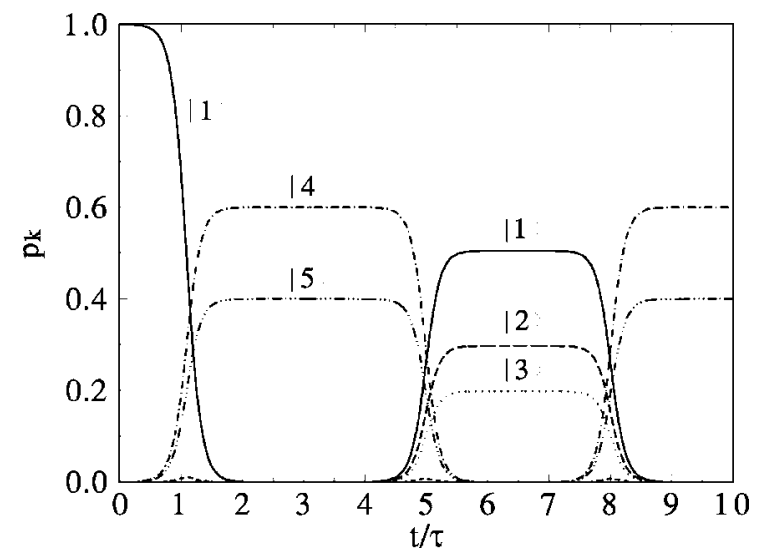

FIG. 6. The time evolution of the $p_{k}=\left|c_{k}\right|^{2}$ populations of $|k\rangle$ $(k=1-5)$ energy eigenstates realized in the chain of transfer processes described in the text.

Rabi frequencies (assumed real for simplicity here) do not cause off-resonant transitions but large enough to satisfy the global adiabaticity condition given in Appendix C. Notice that the wave packets that are associated with those fields are such that their vectors of timedependent coefficients are parallel to the vectors of Rabi frequencies, i.e., $\quad \mathbf{c}^{i}(t) \equiv\left(c_{1}^{i}, \ldots, c_{n}^{i}\right)^{\mathrm{T}}=\cos \theta(t) \mathbf{c}_{0}^{i} \propto \Omega^{i}(t)$ $\equiv\left(\Omega_{1,0}, \ldots, \Omega_{n, 0}\right)^{\mathrm{T}}$ and $\mathbf{c}^{f}(t) \equiv\left(c_{n+1}^{f}, \ldots, c_{n+m}^{f}\right)^{\mathrm{T}}=\sin \theta(t) \mathbf{c}_{0}^{f}$ $\propto \Omega^{f}(t) \equiv\left(\Omega_{n+1,0}, \ldots, \Omega_{n+m, 0}\right)^{\mathrm{T}}$, and their sizes are the same as in Eq. (12).

Figure 6 illustrates the validity of Eq. (18) for the following chain of population transfers: $|1\rangle \rightarrow c_{4}|4\rangle+c_{5}|5\rangle$ $\rightarrow c_{1}|1\rangle+c_{2}|2\rangle+c_{3}|3\rangle \rightarrow c_{4}^{\prime}|4\rangle+c_{5}^{\prime}|5\rangle$. In the first link of the transfer chain, $|1\rangle \rightarrow c_{4}|4\rangle+c_{5}|5\rangle$, which is seen to exhibit $100 \%$ transfer probability, the multimode Rabi frequencies were chosen as $\Omega_{0,1}(t)=\mathcal{O}_{0,1} \exp \left[-\left(t-t_{0}\right)^{2} / \tau^{2}\right]$ and $\Omega_{0, \nu}(t)=\mathcal{O}_{0, \nu} \exp \left[-t^{2} / \tau^{2}\right](\nu=4,5)$, with $t_{0}=2 \tau$ being the delay between pulses. The coefficients of Eq. (19) are chosen to be $\mathcal{C}=\mathcal{C}^{\prime}=100 / \tau$. The next transfer link is realized by repeating the pulses with different central times and amplitudes $\mathcal{O}_{0, k}$, in accordance with Eq. (19).

We now return to the INS's and FNS's defined in Eq. (17). It is of particular importance to note that these states, which are orthogonal to the initially populated $\left|\Psi^{i}\left(t_{\text {in }}\right)\right\rangle=\Sigma_{k} c_{k}^{i}\left(t_{\text {in }}\right) e^{-i \omega_{k} t_{\text {in }}}|k\rangle$ state and to the finally populated $\left|\Psi^{f}\left(t_{\text {end }}\right)\right\rangle=\Sigma_{l} c_{l}^{f}\left(t_{\text {end }}\right) e^{-i \omega l l_{\text {end }}}|l\rangle$ state, are not adiabatically coupled to the transferring dark state, as we show in Appendix B. Therefore, the initial population evolves from $\left|\Psi^{i}\left(t_{\text {in }}\right)\right\rangle$ to $\left|\Psi^{f}\left(t_{\text {end }}\right)\right\rangle$ unperturbed by the dark INS's and FNS's.

\section{QUANTUM CONTROL PROBLEM IN THE PRESENCE OF DEGENERATE STATES}

In the degenerate quantum control (DQC) problem the goal is to control population transfer between wave packets with arbitrary expansion coefficients, $\left|\Psi^{i}\left(t_{\text {in }}\right)\right\rangle$

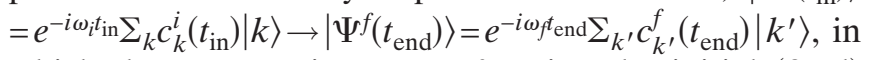
which the energy eigenstates forming the initial (final) wave packets are nearly degenerate, i.e., essentially de-
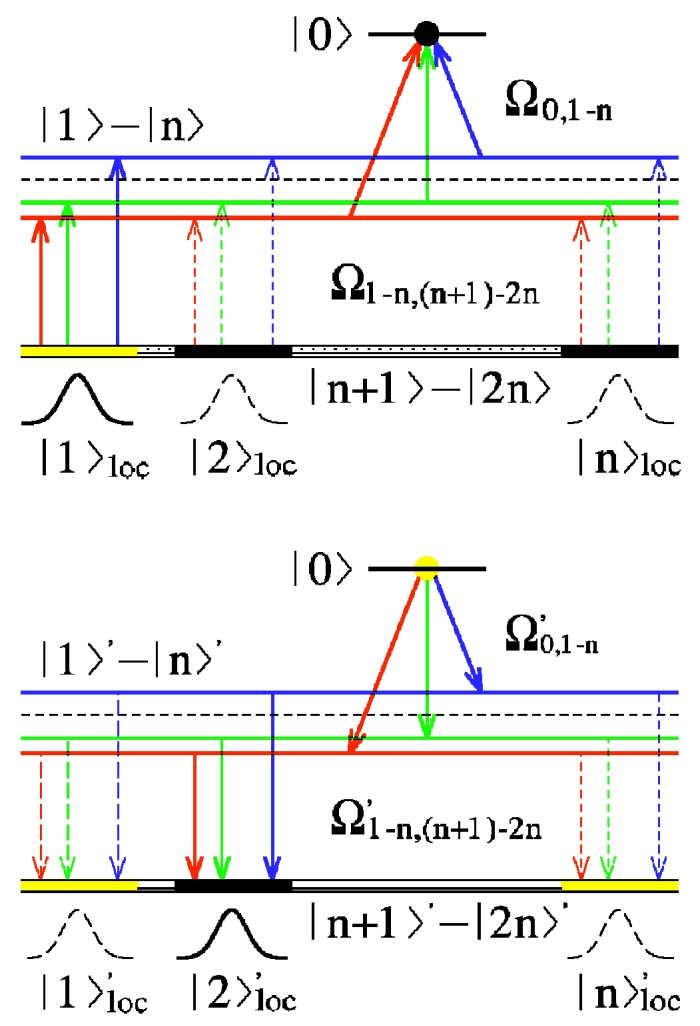

FIG. 7. (Color online) The DQC scheme. (Top) The first step: population in an initial wave packet $\left|\Psi^{i}\right\rangle=|1\rangle_{\text {loc }}$, composed of $n$ nearly degenerate eigenstates, is transferred to a single state $|0\rangle$ by a two-photon adiabatic passage via $n$ nondegenerate intermediate states. (Bottom) The second step: population transfer by a time-reversed process with different Rabi frequencies from $|0\rangle$ to the target wave packet $\left|\Psi^{f}\right\rangle=|2\rangle_{\text {loc }}^{\prime}$, composed of another set of nearly degenerate eigenstates.

generate within the resolution defined by the duration of the pulses used for the transfer. The problem is, thus, more demanding than the nondegenerate one because it is no longer possible to use the different frequency components of the field to address different component states. Addressing the component energy eigenstates in this AP process must therefore be based on phasecontrolled interferences.

In spite of the above difficulty, we have shown (Thanopulos et al., 2004) that when the Rabi-frequency vectors are linearly independent, DQC can be executed by merging the AP and $\mathrm{CC}$ techniques [see explanation below Eq. (22)]. As illustrated in Fig. 7, this is done in two steps: In the first step (upper panel), one uses a pair of laser pulses to adiabatically transfer the population of an initial wave packet $\left|\Psi^{i}\right\rangle$, composed of the nearly degenerate energy eigenstates $|k\rangle(k=n+1, \ldots, 2 n)$, to a single ("parking") state $|0\rangle$, using the nondegenerate auxiliary states $|j\rangle(j=1, \ldots, n)$ as intermediates. As in NQC, the use of nonintuitive pulse ordering guarantees complete population transfer to the parking state $|0\rangle$, which implies that the Stokes pulse, characterized by Rabi frequencies $\Omega_{0 ; 1, \ldots, n}$, linking all $|j\rangle$ states to the $|0\rangle$ state, is made to precede the pump pulse, characterized by $\Omega_{n+1, \ldots, 2 n ; 1, \ldots, n}$ Rabi frequencies, linking all $|k\rangle$ states to 
all $|j\rangle$ states. In the second step (Fig. 7, lower panel), the population in the parking state $|0\rangle$ is transferred to the target wave packet $\left|\Psi^{f}\right\rangle$, composed of the same (or different) set of nearly degenerate eigenstates $\left|k^{\prime}\right\rangle$. The transfer is executed in an adiabatic fashion, with the pump pulse, now linking $|j\rangle$ states and the $|0\rangle$ state, following the Stokes pulse, linking the final $\left|k^{\prime}\right\rangle$ states with all $|j\rangle$ states. We note that using two separate steps, each including a transfer between a set of $n$ degenerate states and a single nondegenerate level via $n$ nondegenerate intermediate states, presents a different approach to the solution of the DQC problem suggested by others (Morris and Shore, 1983; Shore, 1990; Kis et al., 2004), where only one step is considered and degeneracy is allowed within each set of initial, final, and intermediate states.

The electric field of the pump and Stokes pulses used in the first step is $\vec{E}(t)=\vec{E}_{S}(t)+\vec{E}_{P}(t)$, where

$$
\begin{aligned}
& \vec{E}_{S}(t)=\operatorname{Re} \sum_{j=1}^{n} \hat{\epsilon}_{0, j} \mathcal{E}_{0, j}(t) e^{-i \omega_{0, j} t}, \\
& \vec{E}_{P}(t)=\operatorname{Re} \sum_{j=1}^{n} e^{-i \omega_{j, D^{t}}} \sum_{i=n+1}^{2 n} \hat{\epsilon}_{j, i} \mathcal{E}_{j, i}(t) .
\end{aligned}
$$

In the second step the form assumed by the Stokes and pump pulses is being reversed.

The Stokes and pump field components are characterized by the polarization directions $\hat{\epsilon}_{0, j}$ and $\hat{\epsilon}_{j, i}$ and the slowly varying amplitudes $\mathcal{E}_{0, j}(t)$ and $\mathcal{E}_{j, i}(t)$. The center frequencies of the field components are chosen to be in near resonance with the system's transition frequencies, $\omega_{0, j}=\omega_{0}-\omega_{j}$ and $\omega_{j, D}=\omega_{j}-\omega_{D}$, where $\omega_{D}$ is the energy of the nearly degenerate initial (final) states. As in the NQC case, we choose all Rabi frequencies to have a common time envelope, $\Omega_{j, i}(t)=\mathcal{O}_{j, i} f_{\nu}(t)(\nu=S, P)$, with $f_{S}$ preceding $f_{P}$ in both steps. We assume that the conditions imposed on the field amplitudes as discussed in Appendix $\mathrm{C}$ are valid here too.

The vector of coefficients $\mathbf{c}(t)=\left(c_{0}, c_{1}, \ldots, c_{n}\right.$, $\left.c_{n+1}, \ldots, c_{2 n}\right)^{\mathrm{T}}$ in the wave-function expansion of bare states in the interaction representation is obtained by solving Eq. (8), with the RWA Hamiltonian $\mathrm{H}(t)$ written in a compact form including submatrices as

$$
\mathrm{H}(t)=\frac{\hbar}{2}\left(\begin{array}{ccc}
0 & \boldsymbol{\Omega}_{0} & \mathbf{0} \\
\boldsymbol{\Omega}_{0}^{\dagger} & \mathbf{0} & \mathbf{H}_{F} \\
\mathbf{0} & \mathbf{H}_{F}^{\dagger} & \mathbf{0}
\end{array}\right), \quad \mathbf{H}_{F}=\left(\begin{array}{l}
\boldsymbol{\Omega}_{1} \\
\ldots \\
\boldsymbol{\Omega}_{n}
\end{array}\right)
$$

and

$$
\begin{aligned}
& \boldsymbol{\Omega}_{0}=\left(\Omega_{0,1}(t), \ldots, \Omega_{0, n}(t)\right), \\
& \boldsymbol{\Omega}_{j}=\left(\Omega_{j, n+1}(t), \ldots, \Omega_{j, 2 n}(t)\right), \quad j=1, \ldots, n,
\end{aligned}
$$

with $\dagger$ denoting the Hermitian conjugation operation. The $\boldsymbol{\Omega}_{0, \ldots, n}$ vectors of time-dependent Rabi frequencies are different in the two steps because they control the population transfer between different wave packets.

We now show that in order to address arbitrary wave packets in any DQC step, the corresponding Rabi- frequency vectors $\left\{\boldsymbol{\Omega}_{1}, \ldots, \boldsymbol{\Omega}_{n}\right\}$ must be linearly independent of one another. When linear independence is assured, $\mathcal{D} \equiv \operatorname{det}\left(\mathbf{H}_{F}\right) \neq 0$ and the Hamiltonian $\mathrm{H}(t)$ has just one zero eigenvalue, with the corresponding null eigenvalue vector being given as $\mathbf{c}=(\mathbf{1}, \mathbf{0}, \mathbf{x})^{\mathrm{T}}$, where $\mathbf{0}$ denotes an $n$-dimensional zero vector and $\mathbf{x}$ is an $n$-dimensional vector given as $\mathbf{x}=-\mathbf{H}_{F}^{-1} \Omega_{0}^{\dagger}$. Direct operation of $\mathrm{H}(t)$ on $\mathbf{c}$ then confirms that this is the null eigenvalue state.

By constructing the $\Omega_{0}^{\dagger}$ vector of Eq. (22) to be proportional to the $k$ th column of the $\mathbf{H}_{F}$ matrix, i.e., $\Omega_{0, i}^{*}$ $\propto \Omega_{i, k}(k=1, \ldots, n)$, we guarantee that the $\mathbf{c}$ vector correlates at the start of the first step (end of the second step) with $|k\rangle\left(\left|k^{\prime}\right\rangle\right)$ states and at the end of the first step (the start of the second step) with the $|0\rangle$ state. Hence, it follows from the linearity of the above equations that with the choice $\Omega_{0, i}^{*} \propto \sum_{k=1}^{n} c_{k} \Omega_{i, k}$, where $c_{k}=c_{k}^{i}\left(c_{k}^{f}\right)$ at the start of the first step (end of the second step), the null eigenvalue state correlates at the start of the first step (end of the second step) with the $\left|\Psi^{i}\right\rangle\left(\left|\Psi^{f}\right\rangle\right)$ superposition state. The efficiency of the two DQC steps also does not suffer from the nonorthogonality of the linearly independent $\boldsymbol{\Omega}_{j}(j=1, \ldots, n)$ vectors (Thanopulos et al., 2004). Thus, complete control over the population transfer between arbitrary wave packets, composed of nearly degenerate states, can in principle be achieved.

As we show below, even if all $i=n+1, \ldots, 2 n$ states are degenerate, it is possible to guarantee the linear independence of the Rabi-frequency vectors. Such linear independence may be achieved if the quasidegenerate states are coupled in such a way that each Rabi frequency has a different (complex) phase. The linear independence of the Rabi-frequency vectors is achieved by combining the properties of the states and their dipole elements with the degrees of freedom possessed by the light beams, i.e., their frequencies, phases, and polarizations. In the $\mathrm{CC}$ of current directionality, discussed in Sec. II.A, linear independence is achieved with different phases of the light beams used. In the control of the $\mathrm{Al}_{3} \mathrm{O}$ isomerization discussed below, linear independence is achieved if the dipole-moment vectors $\vec{\mu}_{j, i}$ point in different directions, so that independent polarizationvector $\hat{\epsilon}_{j, i}$ directions can be used. Obviously, only a limited number of degrees of freedom can be gained in this way, so that other degrees of freedom, mostly based on the character of excited states, must be used in systems with more degenerate levels.

We now illustrate the versatility of the above DQC method by applying it to the control of isomerization processes of the Jahn-Teller distorted $\mathrm{Al}_{3} \mathrm{O}$ molecule (Bunker and Jensen, 1998). As shown in Fig. 8, the JahnTeller effect results in the formation of three identical broken-symmetry minima (isomers) corresponding to T-shaped configurations in which the distance between two aluminum atoms is smaller than their distance to the third atom (Boldyrev and von R. Schleyer, 1991). This situation gives rise to three sets of highly stable wave packets localized about each T-shaped minimum. The in-plane tunneling motion of a localized wave packet from one such minimum to another is known as pseu- 


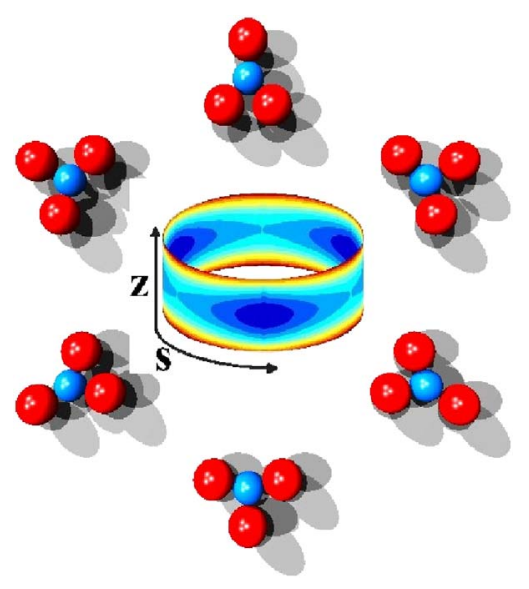

FIG. 8. (Color online) The three Jahn-Teller-distorted stable T-shaped configurations of the $\mathrm{Al}_{3} \mathrm{O}$ molecule and the three equilateral transition-state saddle points which separate them. In the center, we show the potential along $s$-the pseudorotation-and $z$-the out-of-plane-motion of $\mathrm{O}$ relative to the $\mathrm{Al}_{3}$ plane.

dorotation. Another important motion is the out-ofplane vibration of the oxygen atom relative to the $\mathrm{Al}_{3}$ triangle.

Our control objective is to transfer population between localized states of the three minima as completely as possible. The control objective falls under the DQC category because low-lying localized states appear in triplets, which are nearly threefold degenerate, as we discuss below. To simplify the dynamics, we consider partial or complete hindering of the overall rotation of the molecule by, e.g., depositing it on a surface of some material or in a pocket of a larger molecule. As a result, the rotation levels become highly separated and can be neglected in the present analysis. Then the in-plane $(x-y)$ and out-of-plane $(z)$ motions occur in well-defined laboratory directions that are parallel and perpendicular to the surface, respectively. This allows us to choose the polarization directions of the field components that will couple with dipole-moment components that are linearly independent. As shown below, in order to do that we need to use states in which one of the out-of-plane vibrational modes is excited (Thanopulos et al., 2004).

Our simulation of the control dynamics proceeds by first obtaining, using ab initio methods (Frisch et al., 2001), the potential surface and electric dipole moments in a two-dimensional (2D) subspace of the full (6D) configuration space of $\mathrm{Al}_{3} \mathrm{O}$ containing the pseudorotation $(s)$ and out-of-plane $(z)$ modes (see Fig. 8). The relevant eigenstates and eigenenergies are obtained using wellestablished methodologies (Mayer and Günthard, 1968, 1970; Hartchcock and Laane, 1985) for solving the 2D nuclear Schrödinger equation $\left[E_{k}-H\right] \phi_{k}(s, z)=0$.

The conical intersection with the first excited state occurs at sufficiently high energies (Boldyrev and von R. Schleyer, 1991) to allow neglect of Berry-phase effects (Berry, 1984; Mead, 1992; Yarkony, 1996, 2001) for relatively low-lying vibrational states (Kendrick, 1997), as used here. As a result, the eigenenergies of our 2D (c)

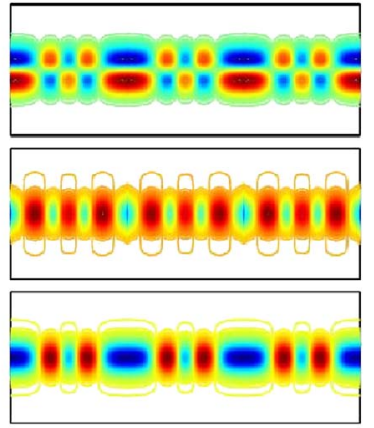

(a)

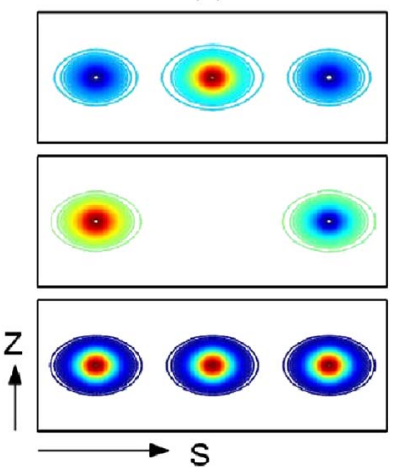

$S$ (d)

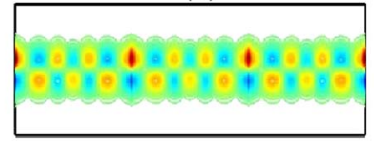

(e)

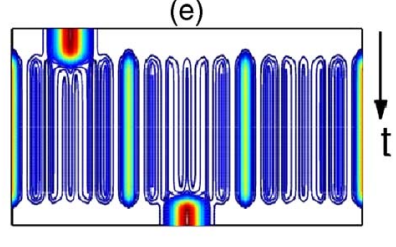

(b)

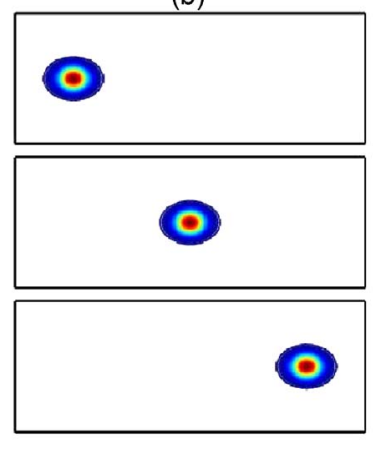

FIG. 9. (Color online) The DQC dynamics: (a) The three lowest $\{0,0\}$ energy eigenstates of $\mathrm{Al}_{3} \mathrm{O}$ as a function of the $s$ and $z$ coordinates. (b) Superpositions of these eigenstates form localized wave packets about each T-shaped minimum. (c) The $|j\rangle$ intermediate states. (d) The $|0\rangle$ parking state. (e) The time dependence of the DQC dynamics, showing controlled transfer between two T-shaped minima.

model are describable by two $\left\{v_{s}, v_{z}\right\}$ quantum numbers, essentially enumerating the number of nodes along the $s$ and $z$ directions in each T-shaped minimum (see also Fig. 9). The low-lying $\left\{v_{s}, v_{z}\right\}$ states appear as triplets, each triplet composed of two degenerate eigenstates and a third, nondegenerate, state.

The three eigenstates in the lowest $(\{0,0\})$ triplet are shown in Fig. 9(a). We take linear combinations of these states to form the initial (final) localized wave packets $\left|\Psi^{\alpha}\right\rangle=\Sigma_{k} c_{k}^{\alpha}|k\rangle(\alpha=i, f, i, f=1,2,3)$, displayed in Fig. 9(b). The three nondegenerate delocalized components of the $\{4,0\},\{6,0\}$, and $\{4,1\}$ triplets, shown in Fig. 9(c), are chosen as the $|j\rangle$ intermediate states. Finally, the nondegenerate component of the $\{6,1\}$ triplet, presented in Fig. 9(d), is used as the parking state $|0\rangle$.

The three nondegenerate intermediate states are coupled to the localized states $\left|\Psi^{i}\right\rangle$ by transitions along the $x, y$, and $z$ directions, respectively. The same states are coupled to the parking $|0\rangle$ state by transitions along the $z, z$, and $y(x)$ directions, respectively. In this way we are able to achieve the required linear independence of the $\boldsymbol{\Omega}_{1}, \boldsymbol{\Omega}_{2}$, and $\boldsymbol{\Omega}_{3}$ Rabi-frequency vectors, due to the fact that the corresponding dipole matrix elements form linearly independent vectors.

We now need to write the Rabi frequencies for the two steps, each of which contains two $(S$ and $P$ ) pulses. 
We can combine the Rabi frequencies for both DQC steps in the following expressions having all four pulses:

$$
\begin{aligned}
\Omega_{j, k}(t)= & \Omega_{j, k}^{\max }\left\{\exp \left[-(t+\tau)^{2} / \tau^{2}\right]+\exp \left[-(t-\tau)^{2} / \tau^{2}\right]\right\}, \\
\Omega_{k, 0}(t)= & \Omega_{k, 0}^{\max }\left\{\exp \left[-(t+3 \tau)^{2} / \tau^{2}\right]\right. \\
& \left.+\exp \left[-(t-3 \tau)^{2} / \tau^{2}\right]\right\},
\end{aligned}
$$

with $\Omega_{j, k}^{\max }=\Omega_{k, 0}^{\max }=0.4 \mathrm{~cm}^{-1}$ and $\tau=5 \mathrm{~ns}$. The dipolemoment matrix elements are of the order $10^{-3}-10^{-1}$ Debye. The duration of the laser pulses is roughly $8 \tau \ll \tau_{i}$ (Thanopulos et al., 2004), where $\tau_{i}$ is the tunneling time (in the $\mu$ sec time scale according to our calculations) from one minimum to the next, so that the $\left|\Psi^{i}\right\rangle(i=1,2,3)$ states remain localized in their corresponding T-shaped minima for sufficiently long times to be used as legitimate initial and final states.

In Fig. 9(e), we display the time evolution of the system during the two steps of the DQC process. The $z$-integrated probability density $\int d z|\langle s, z \mid \Psi(t)\rangle|^{2}$ is presented as a function of $s$ and $t$. We start with the localized $\left|\Psi^{1}\right\rangle$ state being initially populated. At the end of the first step, its population is parked on the excited delocalized $|0\rangle$ state, using as intermediate the $|j\rangle(j$ $=1,2,3$ ) states (which remain unpopulated throughout the process). In the parking state $|0\rangle$, the probability density is symmetrically concentrated near the three saddle points. The parking time should be short enough so that no population is lost due to relaxation. Finally, at the end of the second step, the parked population is transferred to the localized $\left|\Psi^{2}\right\rangle$ state. Note that the DQC process can be generalized for selective and complete population transfer to several energetically degenerate continuum channels that form the manifold of either the initial or final states (Thanopulos and Shapiro, 2006).

\section{COHERENTLY CONTROLLED QUANTUM ADIABATIC ENCODING AND DECODING}

In addition to quantum control, the systems analyzed above are of interest in the context of quantum encoding and decoding of information (Král and Shapiro, 2004), quantum computation (Nielsen and Chuang, 2000), quantum communication (Duan et al., 2001), and the preparation of various quantum devices (Vandersypen, 2001; Weinstein et al., 2001). We focus on systems involving nondegenerate states here.

Contrary to quantum control, where we wish to transfer population from one known state to another, in the quantum decoding problem we do not know the initial state. Rather we should devise a process that transfers population from any initially populated state that belongs to a set of known (predetermined) states to one of the final energy eigenstates, according to a predetermined protocol. If this population is predominantly transferred to a single eigenstate, we say that the quantum information has been decoded in an adequate manner. This process is in fact a kind of mapping of the initial states on the final states.

In the particular quantum decoder shown in Fig. 10

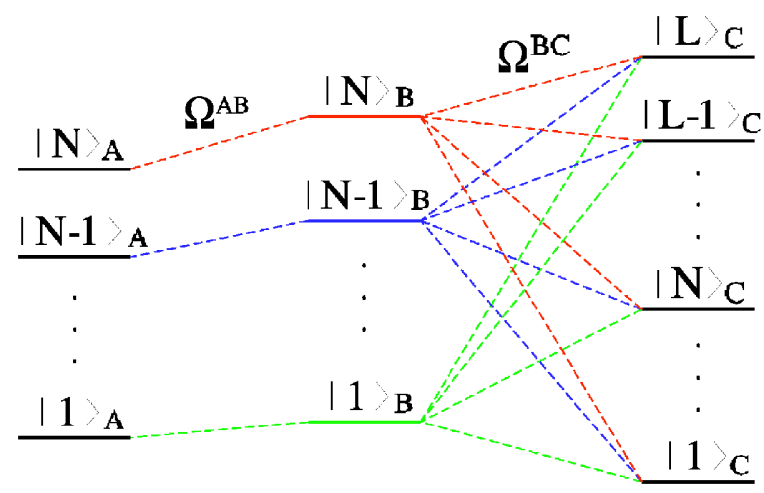

FIG. 10. (Color online) The adiabatic decoder. Quantum information that encoded in the phases of the population amplitudes of the $\left|1_{A}\right\rangle, \ldots,\left|N_{A}\right\rangle$ states is used to transfer the population to predominantly one of the $\left|1_{C}\right\rangle, \ldots,\left|L_{C}\right\rangle$ states.

(Král and Shapiro, 2004), information is encoded on a superposition of $N$ energy eigenstates, $\left|i_{A}\right\rangle(i=1, \ldots, N)$, the $M$ distinct phases of whose population amplitudes form a discrete set of "quNits" (Kaszlikowski et al., 2000). (The term "quNits" is the generalization of the term "quBits" to nonbinary codes.) Decoding is realized by transferring, according to a predetermined protocol, the population of each linear combination of initial $\left|i_{A}\right\rangle$ states which represents a number to (predominantly) a single final $\left|k_{C}\right\rangle$ state. The transfer is induced by a combination of a pump pulse, which couples each initial $\left|i_{A}\right\rangle$ state to a unique intermediate state $\left|i_{B}\right\rangle$, and a Stokes pulse, which couples each $\left|i_{B}\right\rangle$ state to all the final $\left|k_{C}\right\rangle$ states $(k=1, \ldots, L$ with $L \geqslant N)$. The Rabi frequency coupling terms associated with the pump pulse are denoted as $\Omega_{i i}^{A B}$, whereas those associated with the Stokes pulse are denoted as $\Omega_{i k}^{B C}$.

As in the AP examples discussed in Secs. III and IV, we use the counterintuitive pulse ordering in which the Stokes pulse precedes the pump pulse. Assuming a single, linear, polarization direction here, the scalar components of the electric fields of the two pulses are given as $E_{P}(t)=\operatorname{Re} \Sigma_{k, l} \mathcal{E}_{k, l}^{A B}(t) e^{-i \omega_{k, l}^{A B} t}$ and $E_{S}(t)$ $=\operatorname{Re} \Sigma_{k, l} \mathcal{E}_{k, l}^{B C}(t) e^{-i \omega_{k, l}^{B C} t}$. Given the field components, the Rabi frequencies are defined as $\Omega_{k l}^{\alpha}(t) \equiv \mu_{k, l}^{\alpha} \mathcal{E}_{k, l}^{\alpha}(t) / \hbar$ $\equiv \mathcal{O}_{k, l}^{\alpha} f_{\nu}(t) \quad(\nu=S, P, \alpha=A B, C D)$, where $\mu_{k, l}^{\alpha}$ are the $\left|k_{A(B)}\right\rangle \leftrightarrow\left|l_{B(C)}\right\rangle$ matrix elements of the projection of the dipole moment on the polarization directions of the fields. As in Secs. III and IV, $0<f_{\nu}(t)<1(\nu=S, P)$ are two time-dependent functions that determine the temporal properties of the Stokes and pump pulses.

The total Hamiltonian is given as

$$
\begin{aligned}
H(t)= & H_{M}+\frac{\hbar}{2} \operatorname{Re} \sum_{i=1}^{N}\left[\Omega_{i i}^{A B}(t) e^{-i \omega_{i, i}^{A B} t}\left|i_{B}\right\rangle\left\langle i_{A}\right|+\text { H.c. }\right] \\
& +\frac{\hbar}{2} \operatorname{Re} \sum_{i=1}^{N} \sum_{k=1}^{L}\left[\Omega_{k i}^{B C}(t) e^{-i \omega_{k, i}^{B C} t}\left|k_{c}\right\rangle\left\langle i_{B}\right|+\text { H.c. }\right] .
\end{aligned}
$$

In the first sum, $\ell$ goes over all states of the system, with 
energies $E_{\ell}$. The second term represents the one-to-one resonant coupling of the initial states to the intermediate states by the pump pulse whose frequency components are $\omega_{i, i}^{A B} \equiv \omega_{i_{B}}-\omega_{i}$. The third term represents the resonant coupling of each intermediate state to every final state by the Stokes pulse, with frequency components $\omega_{k, i}^{B C} \equiv \omega_{k_{C}}-\omega_{i_{B}}$.

The vector of expansion coefficients $\mathbf{c}=\left(c_{1}, c_{2}, \ldots\right)^{\mathrm{T}}$ is obtained by solving the Schrödinger equation (8), with the time-dependent Hamiltonian in the RWA given in compact form as

$$
\mathbf{H}(t)=\frac{1}{2}\left(\begin{array}{ccc}
\mathbf{0} & \mathbf{H}^{A B^{\dagger}} & \mathbf{0} \\
\mathbf{H}^{A B} & \mathbf{0} & \mathbf{H}^{B C \dagger} \\
\mathbf{0} & \mathbf{H}^{B C} & \mathbf{0}
\end{array}\right),
$$

where $\mathbf{H}_{i, j}^{A B}=\hbar \Omega_{i i}^{A B} \delta_{i j}$ is a diagonal submatrix and $\mathbf{H}_{i k}^{B C}$ $=\hbar \Omega_{i k}^{B C}$ is a full submatrix. We assume that the level energies are such that only the coupling of the initial states to the intermediate states by the pump pulse whose frequency components are $\omega_{i, i}^{A B} \equiv \omega_{i_{B}}-\omega_{i_{A}}$ and the coupling of each intermediate state to every final state by the Stokes pulse, with frequency components $\omega_{k, i}^{B C} \equiv \omega_{k_{C}}$ $-\omega_{i_{B}}$, are resonant. Side effects due to off-resonant couplings to neighboring states are ignored (Král and Shapiro, 2002). As discussed in Appendix B, we can neglect off-resonance terms under the assumption of sufficiently large level spacing compared to the Rabi frequencies used (Shore, 1990). $\mathrm{H}(t)$ has $2 N+L$ eigenvalues, of which $2 N$ at most are nonzero, $\lambda_{1, \ldots, 2 N}(t) \neq 0$. The remaining $L$ eigenvalues are zero, $\lambda_{2 N+1, \ldots, 2 N+L}=0$, giving rise to dark states.

We can verify from the null eigenvalue state definition $\mathrm{H}(t) \cdot \mathbf{d}_{k}(t)=0(k=1, \ldots, L)$ that the expansion coefficients of the null eigenvalue states $\mathbf{d}_{k}(t)$ are given as

$$
\begin{aligned}
& \mathbf{d}_{1}=\left(\Omega_{11}^{B C} / \Omega_{11}^{A B}, \ldots, \Omega_{N 1}^{B C} / \Omega_{N N}^{A B}, 0, \ldots, 0,-1,0, \ldots, 0\right)^{\mathrm{T}}, \\
& \mathbf{d}_{2}=\left(\Omega_{12}^{B C} / \Omega_{11}^{A B}, \ldots, \Omega_{N 2}^{B C} / \Omega_{N N}^{A B}, 0, \ldots, 0,0,-1, \ldots, 0\right)^{\mathrm{T}}, \\
& \ldots \\
& \mathbf{d}_{L}=\left(\Omega_{1 L}^{B C} / \Omega_{11}^{A B}, \ldots, \Omega_{N L}^{B C} / \Omega_{N N}^{A B}, 0, \ldots, 0,0,0, \ldots,-1\right)^{\mathrm{T}} .
\end{aligned}
$$

In order to simplify the analysis, we assume, without loss of generality (Král et al., 2002), that $\Omega_{11}^{A B}=\Omega_{22}^{A B}=\cdots$ $=\Omega_{N N}^{A B}=\Omega^{A B}$. With this simplification, the first $N$ coefficients in the $\mathbf{d}_{k}(t)$ null eigenvectors are solely determined by the vector of the Stokes-field Rabi frequencies $\boldsymbol{\Omega}_{S k}=\left(\Omega_{1 k}^{B C}, \Omega_{2 k}^{B C}, \ldots, \Omega_{N k}^{B C}\right)^{\mathrm{T}}$. Moreover, the $\mathbf{d}_{k}(t)$ states correlate on a one-to-one basis with the $\left|1_{C}\right\rangle, \ldots,\left|\mathbf{L}_{C}\right\rangle$ final states. Thus, if we manage to initially populate only one of the $\mathbf{d}_{k}(t)$ states, AP would exclusively transfer the population to a single final $\left|k_{C}\right\rangle$ state at the end of the process.

If the transfer is exclusively to a single state, the decoding process is perfect. If the transfer is nonexclusive, but still predominantly to a single target state, we deem the decoding, though not perfect, adequate. This is the case in most of the trial cases we ran: It is not possible

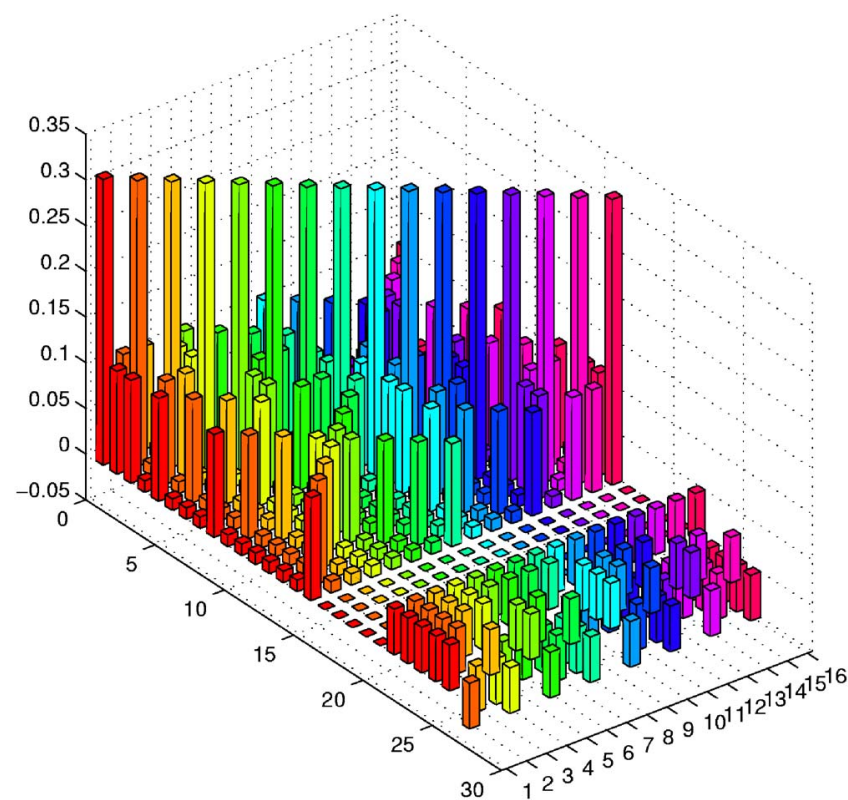

FIG. 11. (Color online) The results of the binary-tohexadecimal decoder for differently encoded information on the $N=5$ initial states (front). In the back, we see the populations of the $2^{N-1}=16$ final levels for these cases, while the intermediate states, in the midregion, are unpopulated.

to populate just a single dark state. In fact, the initial population of the $\left|1_{A}\right\rangle, \ldots,\left|N_{A}\right\rangle$ states is distributed among several $(k=1, \ldots, L)$ null eigenvalue states. The distribution $p_{k}$ is determined by the square of the projection vector of initial coefficients $\mathbf{c}_{0}=\left(c_{1}, c_{2}, \ldots, c_{N}\right)^{\mathrm{T}}$ on the vector of Stokes-field Rabi frequencies $\boldsymbol{\Omega}_{S k}$, i.e., $p_{k} \propto\left|\mathbf{c}_{0}^{\dagger} \cdot \boldsymbol{\Omega}_{S k}\right|^{2}$.

Using relations analogous to those in Sec. III for determining the fraction of population transferred through a single intermediate state by many pump Rabi frequencies (Král and Shapiro, 2002; Král et al., 2002), we can maximize the population $p_{i}$, transferred to the predetermined $\left|i_{C}\right\rangle$ state, by choosing $\boldsymbol{\Omega}_{S i} \propto \mathbf{c}_{0}$. At the same time, it is in principle possible to minimize transfer to undesired $\left|k_{C}\right\rangle(k \neq i)$ states, by requiring that the $\boldsymbol{\Omega}_{S k}$ and $\boldsymbol{\Omega}_{S i}$ vectors be orthogonal to each other, $\boldsymbol{\Omega}_{S k}^{\dagger} \cdot \boldsymbol{\Omega}_{S i} \approx 0$.

Whereas the first of the above conditions is easily satisfied, the second condition cannot be fully realized if $N<L$ because we cannot orthogonalize all $\mathbf{L}$ vectors $\boldsymbol{\Omega}_{S k}$ in an $N-(<L)$ dimensional space. Only if $N=L$ can their components be chosen such that all $L$ null eigenvalue states of Eq. (25) will be mutually orthogonal. In that case transfers to the final $\left|k_{C}\right\rangle$ states are both complete and exclusive. The results show that unless $N \ll L$, in most cases adequate, though not perfect, decoding can be realized. In fact, the decoding efficiency can be further increased by cross-correlating the populations of other states, since their populations are different for each input states (see Fig. 11).

As an example, we examine the transfer of numeric information stored in $M=2$ phases of the population coefficients of $N$ equally populated initial levels $\left|i_{A}\right\rangle$. In this 
phase encoding, the binary number $0=(0,0, \ldots, 0)$, i.e., $0 \times 2^{0}+0 \times 2^{1}+\cdots+0 \times 2^{N}$, is represented by the vector of amplitudes $\mathbf{c}_{1}=(1,1, \ldots, 1)$ of $\left|i_{A}\right\rangle$ states; the binary number $(1,0, \ldots, 0)$, i.e., $1 \times 2^{0}+0 \times 2^{1}+\cdots+0 \times 2^{N}=1$, by $\mathbf{c}_{2}=(-1,1, \ldots, 1)$, and so on. Because quantum states are known up to an overall phase, we can, using $N$ levels, encode in this manner only $2^{N-1}$ numbers.

In Fig. 11, we present the adiabatic decoder. The magnitudes of all Rabi frequencies are $\left|\mathcal{O}_{i j}\right|=60 / \tau$, where $f_{\nu}(t)=\exp \left[-\left(t-t_{\nu}\right)^{2} / \tau^{2}\right](\nu=S, P)$, and $t_{P}-t_{S}=2 \tau$. The system decodes each binary-phase stored number by transferring the population of the initial superposition state, representing this number, to predominantly one of the 16 final $\left|k_{C}\right\rangle$ eigenstates. The degree of exclusivity of the transfer is such that five other final states end up with 2.78 times less population, while the remaining ten states being considerably less populated.

We obtain analytically (Král and Shapiro, 2004) the populations $p_{k}$ of the final $\left|k_{C}\right\rangle$ states using the fact that $p_{k} \propto\left|\mathbf{c}_{0}^{\dagger} \cdot \boldsymbol{\Omega}_{S k}\right|^{2}$, where $\mathbf{c}_{0}$ is the encoded initial vector $\mathbf{c}_{0}=\left(c_{1}, c_{2}, \ldots, c_{5}\right)$ and $\boldsymbol{\Omega}_{S k}$ is the Stokes-Rabi-frequency vector. The density of encoding (Braunstein and Kimble, 2000) could be increased by using higher quNit states, obtained by using $M>2$ roots of the identity $\exp (i 2 \pi j / M)$.

The same methodology used in decoding works equally well for encoding. The encoding process can be phrased as starting with a single state and transferring its population according to some preset protocol to a linear combination of other states. The method we have chosen for encoding, which is to time reverse the decoding pulse sequence, has been found to be very effective (Král and Shapiro, 2004) in that the initial-state population is predominantly deposited in the encoded states. The scheme appears to behave like a complex but passive transmissive system.

\section{PURIFICATION OF MIXTURES OF RIGHT-HANDED AND LEFT-HANDED CHIRAL MOLECULES}

A striking example of CCAP (Král and Shapiro, 2001; Král et al., 2003; Thanopulos et al., 2003) is the combination of a variation of the one-photon versus twophoton CC scenario, discussed in Sec. II.A, and AP, discussed in Sec. II.B, to achieve purification of mixtures of enantiomers (Bodenhöfer et al., 1997; McKendry et al., 1998; Quack, 1989; Knowles, 2002; Zepik et al., 2002).

Enantiomers are different stereoconformers of a chiral molecule, which can be discriminated by their ability to rotate the plane of polarization of light in opposite directions (optical rotatory power). However, this effect cannot be used to separate left-handed from righthanded enantiomers in a mixture (racemic mixture) because the number of molecules excited using linear or circularly polarized light is the same for both species.

Coherent control and orientational methods for affecting such separation, or actively converting one enantiomer to the other, have been suggested (Shapiro and Brumer, 1991; Salam and Meath, 1998; Fijimura et al.,
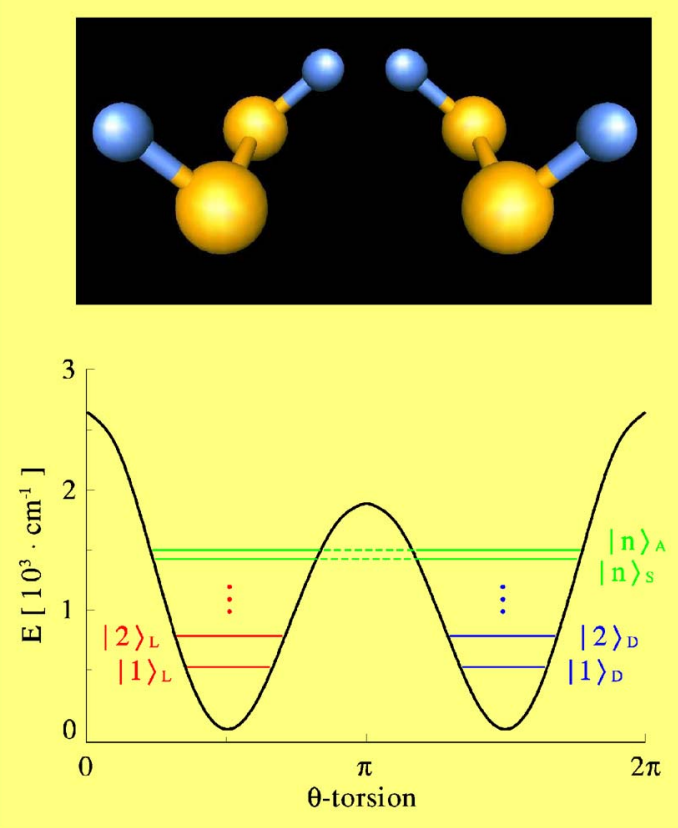

FIG. 12. (Color online) The double-well potential energy curve for the torsional motion of the $\mathrm{D}_{2} \mathrm{~S}_{2}$ molecule. The two enantiomers connected by this stereomutation torsional motion are shown in the upper panel.

1999; Shapiro et al., 2000; Gerbasi et al., 2001; Frishman et al., 2003). In particular, it has been shown that the task can be realized by the so-called laser distillation method (Shapiro et al., 2000; Frishman et al., 2003), which is based on repetitive use of one-photon versus twophoton pathways to the same final state. As in the control of photocurrent directionality discussed in Sec. II.A, the interference between these two pathways can, depending on the relative phases between the three laser fields now used, result in the preferential excitation of one enantiomer (shown as one localized minimum in Fig. 12) relative to the other (residing in the other local minimum). The excited states (shown as delocalized states in Fig. 12) eventually relax back to the two enantiomers. Although this relaxation does not discriminate between the right-handed and left-handed enantiomers, the net result of each excitation-relaxation cycle is to transfer population from the enantiomer which is more readily excited in the first step to the other. Thus, the identity of the enantiomer to be depleted depends on the relative phases of the three laser fields used. The laser distillation process can be repeated until satisfactory conversion is reached.

The laser distillation method, though proved to be very effective for several molecules (Gerbasi et al., 2001; Frishman et al., 2003), requires the continuous application of many laser pulses. Therefore, it is of a principle interest to see whether one could convert one enantiomer to another by using AP methods and applying a certain pulse sequence only once. As discussed in Sec. II.B, ordinary STIRAP is only sensitive to the energy levels and magnitudes of transition-dipole matrix ele- 

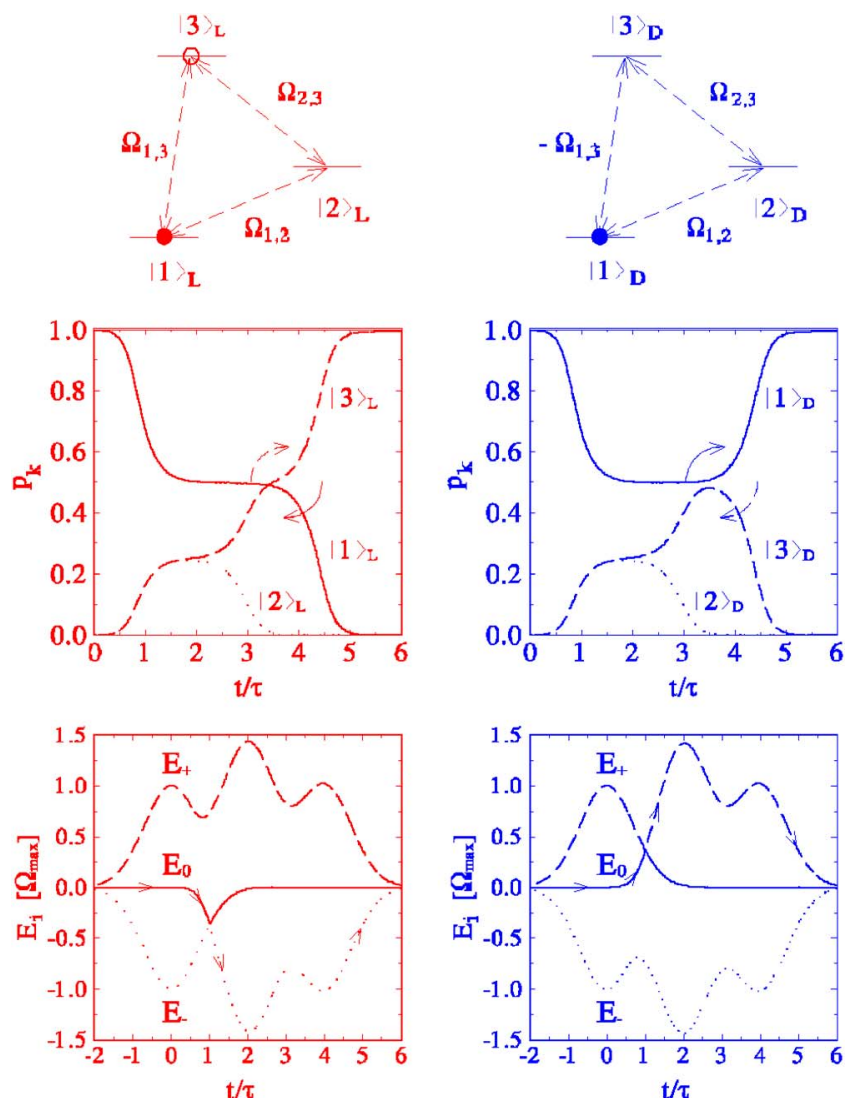

FIG. 13. (Color online) The enantiodiscriminator. (Top) A schematic plot of the CPT scheme, in which three levels of each enantiomer are resonantly coupled by three fields, as used in the discrimination step. (Middle) The time evolution of population of three levels: Both enantiomers start in the $|1\rangle$ state. At the end of the process the $L$ enantiomer is transferred to the $\left|3_{L}\right\rangle$ state, while the $D$ enantiomer remains in the initial $\left|1_{D}\right\rangle$ state. (Bottom) The time dependence of the eigenvalues of the Hamiltonian of Eq. (27): The population initially follows the $\left|E_{0}\right\rangle \equiv\left|\lambda_{0}\right\rangle$ null eigenvalue state. At $t \approx \tau$ it crosses over diabatically to $\left|E_{-}\right\rangle \equiv\left|\lambda_{-}\right\rangle$for one enantiomer and to $\left|E_{+}\right\rangle \equiv\left|\lambda_{+}\right\rangle$for the other. $\left(\left|\lambda_{0, \pm}\right\rangle\right.$ are defined in the text.)

ments, attributes which are identical for both enantiomers. As we show below, the electric-dipole transition matrix elements of the two enantiomers differ only in their phases. Since STIRAP is insensitive to this phase, it is incapable of selecting between enantiomers.

In order to generate phase sensitivity in excitation by AP techniques of quantum systems with broken symmetry, such as chiral molecules, we have enriched STIRAP by one more excitation transfer and termed the resulting scheme cyclic population transfer (CPT) (Král and Shapiro, 2001). In CPT the two-photon pathway $|1\rangle \leftrightarrow|2\rangle \leftrightarrow|3\rangle$ of the STIRAP $\Lambda$ system is supplemented by a one-photon process $|1\rangle \leftrightarrow|3\rangle$ that directly connects the initial and final states around a loop (cycle) (see upper panel of Fig. 13). Although closed-loop systems involving three states have been studied previously (Unanyan et al., 1997; Fleischhauer et al., 1999), our proposal is the first involving levels that any pair of them can be directly coupled by a one-photon process.
The existence of CPT is impossible for molecules with a well-defined parity (i.e., achiral molecules) since two states which are coupled radiatively by a one-photon process cannot be coupled by a two-photon process. This is because parity considerations dictate that the two states connected by a one-photon process must have opposite parities, whereas the same two states, if connected by a two-photon process, must have the same parities. These two conditions cannot coexist; hence, the two processes cannot couple the same two states.

It is worthwhile noting that in the experimental control of current directionality discussed in Sec. II.A the one-photon and two-photon processes excite two different final degenerate continuum states possessing opposite parities. These states then combine to form degenerate Bloch states $\left| \pm \mathbf{k}_{c}\right\rangle$. Their different excitation, signalizing the presence of a current, is controlled by interferences, depending on the phases of the light fields. The interference in that case only affects the directionality of the current but not the total population excited to the continuum. Indeed, when averaging over angles is performed, the one-photon-two-photon interference term vanishes. One can also see the process as a direct, but different excitation of the broken-symmetry states $\left| \pm \mathbf{k}_{c}\right\rangle$ that have ill-defined parities and can be excited by both one-photon and two-photon processes. This provides the linkage to CPT, where discrete states with ill-defined parities are excited analogously by pulses.

In chiral molecules, which per definition lack an inversion center because they exist in broken-symmetry states which have ill-defined parities, such dual coupling is permissible and CPT is possible (Král and Shapiro, 2001; Král et al., 2003; Thanopulos et al., 2003). If we ignore the parity-violating weak interactions in nuclei, whose effect is minuscule, the true eigenstates of chiral molecules do have well-defined parities, but these are not the states the molecules get trapped in. The molecule rather exists in one of the broken-symmetry states localized about either one of the minima of Fig. 12. Each of these states is a linear combination of two eigenstates of opposite parities, which due to the height of the barrier for interconversion between enantiomers are practically degenerate. Hence, the above parity restrictions do not apply in this case.

As depicted in Fig. 13 (top), CPT is driven by three laser fields. The interference between the one-photon and two-photon pathways shown renders the evolution of the system dependent on the total phase $\varphi$ of the three (material+optical) coupling terms. Since the phases of the transition dipoles in the two enantiomers differ by $\pi$ [see Eq. (26) below], the evolution of the two enantiomers under the action of the three fields is different.

In order to demonstrate the method, we now discuss explicitly the purification of a racemic mixture of the (transiently chiral) $\mathrm{D}_{2} \mathrm{~S}_{2}$ molecule (shown schematically in Fig. 12). In this molecule, the pathway connecting the two enantiomers involves a relatively simple motion: that of the (hindered) rotation (torsion) of the D atoms 
about the S-S bond. The tunneling splitting of the lowest torsional states gives enantiomeric lifetimes of several msec (Gottselig et al., 2000; Král et al., 2003; Thanopulos et al., 2003). Thus, although $\mathrm{D}_{2} \mathrm{~S}_{2}$ is only transiently chiral, its chiral molecular structures remain stable for sufficiently long times to perform the excitation process.

According to this proposal, we can achieve chiral purification of the racemic mixture of chiral $\mathrm{D}_{2} \mathrm{~S}_{2}$ molecules in just two steps: In the first discrimination step, based on the CPT process, we excite one enantiomer, while leaving the other one in its initial state. In the second conversion step, we convert the enantiomer excited in the first step to its mirror-imaged form. Because of the completeness of the AP processes used in both steps, the racemic mixture of chiral molecules should be turned into a sample containing the enantiomer of our choice only, after the first-time completion of the two steps.

The two-step enantiopurification process makes use of five pairs of $\mathrm{D}_{2} \mathrm{~S}_{2}$ rovibrational eigenstates. These vibrational states correspond to the combined torsional and S-D asymmetric stretching modes. The wave functions and energies of these states were calculated using potential and electric-dipole surfaces obtained with $a b$ initio methods (Frisch et al., 2001). The rotational states correspond to a rigid rotor. Within the pairs used, ${ }^{2}$ each eigenstate has an $S / A$ (symmetric/antisymmetric) label denoting its parity.

The broken-symmetry states, denoted as $\left|k_{L, D}\right\rangle$, are linear combinations of the essentially degenerate lowerlying symmetric and antisymmetric eigenstates (Quack, 1989), $\left|k_{L, D}\right\rangle=\frac{1}{\sqrt{2}}\left(\left|k_{S}\right\rangle_{ \pm}\left|k_{A}\right\rangle\right), k=1, \ldots, 4$. The higher-lying $\left|5_{S}\right\rangle$ and $\left|5_{A}\right\rangle$ states are already split by $\Delta E_{S, A}^{5}$ $=0.38 \mathrm{~cm}^{-1}$ and can be separately addressed using nsec laser pulses. This cannot be done for the essentially degenerate lower-lying $k=1, \ldots, 4$ eigenstates, for which the splittings are much smaller than the bandwidths of most lasers in practical use.

As in Secs. III and IV, the energy of state $|i\rangle$ is denoted by $E_{i}$, and we choose the external electric field as a sum of components, each being in resonance with one of the $|i\rangle \leftrightarrow|j\rangle$ transition frequencies of interest, $\vec{E}(t)$ $=\operatorname{Re} \Sigma_{i \neq j} \hat{\epsilon} \mathcal{E}_{i, j}(t) e^{-i \omega_{i, j} t}$, where $\omega_{i, j}=\omega_{i}-\omega_{j}$ and $\hat{\epsilon}$ is the polarization direction. The total Hamiltonian has the same form as in Eq. (15).

The control scheme for the three-level discrimination step is shown in the upper panel of Fig. 13. We assume that at sufficiently low temperature the $\mathrm{D}_{2} \mathrm{~S}_{2}$ molecules in the racemic mixture reside initially in the ground $\left|1_{L}\right\rangle$ and $\left|1_{D}\right\rangle$ states. Therefore, our objective at this step is to excite only one enantiomer, i.e., transfer its population completely from the $|1\rangle$ to the $|3\rangle$ state and keep the other in the $|1\rangle$ state (Král and Shapiro, 2001). Due to

\footnotetext{
${ }^{2}$ The $|1\rangle,|3\rangle,|4\rangle$, and $|5\rangle$ states correspond to torsional states with $0,2,3$, and 5 vibrational quanta, respectively. The $|2\rangle$ state is the first excited state of the asymmetric S-D stretching mode.
}

the essential degeneracy of the $\left|i_{L}\right\rangle$ and the $\left|i_{D}\right\rangle$ states for $i=1,2,3$, the $\vec{E}(t)$ field excites simultaneously the $\left|i_{\nu}\right\rangle \leftrightarrow\left|j_{\nu}\right\rangle(i \neq j=1,2,3, \nu=L, D)$ transitions of both enantiomers (Shapiro et al., 2000; Frishman et al., 2003). Therefore, we have to make use of the phase dependence of the CPT process. Using the fact that the electric dipole operator can only connect states of opposite parities, we obtain that

$$
\Omega_{i j}^{L, D}= \pm\left[\left\langle i_{S}|\mu| j_{A}\right\rangle+\left\langle i_{A}|\mu| j_{S}\right\rangle\right] \mathcal{E}_{i j} / \hbar
$$

Hence, all the Rabi frequencies associated with the two enantiomers differ by a sign, i.e., a phase factor of $\pi$, for transitions induced by the dipole component along the stereomutation coordinate. Since in the CPT processes the two enantiomers are influenced by the phase $\varphi^{L, D}$ of the products $\Omega_{12}^{L} \Omega_{23}^{L} \Omega_{31}^{L}$ and $\Omega_{12}^{D} \Omega_{23}^{D} \Omega_{31}^{D}$, we have that $\varphi^{L}-\varphi^{D}=\pi$. This property is invariant to any arbitrary phase change in the individual wave functions of the states $\left|i_{L, D}\right\rangle$.

The vector component of the coefficients c $=\left(c_{1}, c_{2}, c_{3}\right)^{\mathrm{T}}$ of the wave-function expansion for the discrimination step is the solution of the Schrödinger equation (8) with the RWA Hamiltonian after neglecting offresonant terms given as

$$
\mathrm{H}(t)=\frac{\hbar}{2}\left[\begin{array}{ccc}
0 & \Omega_{1,2}^{*}(t) & \Omega_{1,3}^{*}(t) \\
\Omega_{1,2}(t) & 0 & \Omega_{2,3}^{*}(t) \\
\Omega_{1,3}(t) & \Omega_{2,3}(t) & 0
\end{array}\right] .
$$

The phases of the complex Rabi frequencies $\Omega_{i, j}(t)$ are given as $\phi_{i, j}=\phi_{i, j}^{\mu}+\phi_{i, j}^{E}$, where $\phi_{i, j}^{\mu}$ are the phases of the dipole matrix elements $\mu_{i, j}$ and $\phi_{i, j}^{E}$ are the phases of the electric field components $\mathcal{E}_{i, j}$. The evolution of the system is determined by the total phase $\varphi \equiv \phi_{1,2}+\phi_{2,3}$ $+\phi_{3,1}$. This is most noticeable at the time $t=\tau$, for which the three Rabi frequencies are equal in magnitude, $\left|\Omega_{1,2}\right|=\left|\Omega_{1,3}\right|=\left|\Omega_{2,3}\right|=\Omega$.

Denoting the adiabatic eigenvalues of the Hamiltonian of Eq. (27) as $\lambda_{-}, \lambda_{0}$, and $\lambda_{+}$, it is easy to show that they undergo exact degeneracies (crossings) at $t=\tau$, with $\lambda_{+}=2 \Omega$ and $\lambda_{-}=\lambda_{0}=2 \Omega \cos (2 \pi / 3)$, for $\varphi=0$, and $\lambda_{-}$ $=-2 \Omega$ and $\lambda_{+}=\lambda_{0}=-2 \Omega \cos (2 \pi / 3)$, for $\varphi=\pi$. Depending on the polarizations of the fields chosen, one Rabi frequency $\Omega_{i, j}$ of the two enantiomers differs by a sign, making $\varphi$ differ by $\pi$ for the two. Therefore, as shown in the lower panel of Fig. 13, the crossing occurs between different eigenvalues for different enantiomers, leading subsequently to their totally different dynamics (see middle panels of Fig. 13).

The overall discrimination step works as follows: We start with a Stokes pulse $\mathcal{E}_{2,3}(t)$ that couples the $|2\rangle$ and |3) states and with Rabi frequency $\Omega_{2,3}(t)=\Omega^{\max } f(t)$, where $\Omega^{\max }=2 \mathrm{~ns}^{-1}$ and $f(t)=\exp \left(-t^{2} / \tau^{2}\right)$. At this stage of the process the entire population resides in the $\left|\lambda_{0}\right\rangle$ adiabatic eigenstate. In the second stage we simultaneously add two pump pulses with Rabi frequencies $\Omega_{1,2}(t)$ $=\Omega_{1,3}(t)=\Omega^{\max } f(t-2 \tau)$ that couple the $|1\rangle \leftrightarrow|2\rangle$ and $|1\rangle \leftrightarrow|3\rangle$ states. We choose the phases of the optical fields 
such that $\varphi=0$ for one enantiomer and, inevitably, $\varphi=\pi$ for the other. Therefore, the population, which has been following in both enantiomers the initial adiabatic level $\left|\lambda_{0}\right\rangle$, goes at $t=\tau$ smoothly through the crossing region and diabatically transfers to either the $\left|\lambda_{-}\right\rangle$or $\left|\lambda_{+}\right\rangle$state, depending on whether $\varphi=0$ or $\varphi=\pi$, i.e., on the identity of the enantiomer.

After the crossing is complete, at $t>\tau$, the process becomes adiabatic again, with the enantiomer population residing fully in either $\left|\lambda_{-}\right\rangle$or $\left|\lambda_{+}\right\rangle$. At this stage we slowly switch off the $\mathcal{E}_{1,2}(t)$ pulse while making sure that the $\mathcal{E}_{1,3}(t)$ field remains on. This is done by choosing $\Omega_{1,3}(t)=\Omega^{\max }\left(f(t-2 \tau)+f(t-4 \tau) \exp \left\{-i t \Omega^{\max } f(t-6 \tau)\right\}\right)$. As a result, the zero adiabatic eigenstate $\left|\lambda_{0}\right\rangle$ correlates adiabatically with state $|2\rangle$, which thus becomes empty after this process, while the occupied $\left|\lambda_{+}\right\rangle$and $\left|\lambda_{-}\right\rangle$adiabatic states correlate to $\left|\lambda_{ \pm}\right\rangle \rightarrow\left(|1\rangle_{ \pm}|3\rangle\right) / \sqrt{2}$.

The chirp, $\exp \left\{-i t \Omega^{\max } f(t-6 \tau)\right\}$, in the second term of $\Omega_{1,3}(t)$ causes a $\pi / 2$ rotation in the $\{|1\rangle,|3\rangle\}$ subspace at $t \approx 5 \tau$. As a result, state $\left|\lambda_{+}\right\rangle$goes over to state $|3\rangle$ and state $\left|\lambda_{-}\right\rangle$goes over to state $|1\rangle$, or vice versa, depending on $\varphi$. The net result of the adiabatic passage and rotation is that one enantiomer returns to its initial $|1\rangle$ state and the other switches over to the $|3\rangle$ state. As shown in the middle panel of Fig. 13, the enantiodiscriminator is very robust, with all population transfer processes occurring in a smooth fashion.

After the entire population in the $L$ enantiomer has been successfully transferred to the $\left|3_{L}\right\rangle$ state, we use a combination of the solutions of NQC and DQC presented in Secs. III and IV to convert the $\left|3_{L}\right\rangle$ population to the $D$ enantiomer. We do so by performing the $\left|3_{L}\right\rangle$ $\rightarrow\left|4_{D}\right\rangle$ transfer with two in-parallel executed three-level STIRAP processes, using as intermediates the $\left|5_{S}\right\rangle$ and $\left|5_{A}\right\rangle$ states. Since no population is left in state $\left|1_{L}\right\rangle$, the center frequencies of the fields involved are tuned to guarantee that the $D$ enantiomer remains intact in state $\left|1_{D}\right\rangle$.

Schematically, the dynamics follows the $\left|3_{L}\right\rangle \rightarrow \alpha\left|5_{S}\right\rangle$ $+\beta\left|5_{A}\right\rangle \rightarrow\left|4_{D}\right\rangle$ pathway shown in Fig. 14. The transfer is realized by simultaneously introducing two Stokes pulses $\mathcal{E}_{4,5 S}(t)$ and $\mathcal{E}_{4,5 A}(t)$ [of duration $\tau \gg\left(\omega_{5 S}-\omega_{5 A}\right)^{-1}$ ], which resonantly couple each of the $\left|5_{S}\right\rangle$ and $\left|5_{A}\right\rangle$ states to the $\left|4_{L}\right\rangle$ and $\left|4_{D}\right\rangle$ states. After a delay of $2 \tau$, we introduce two pump pulses $\mathcal{E}_{3,5 S}(t)$ and $\mathcal{E}_{3,5 A}(t)$, which resonantly couple each of the $\left|5_{S}\right\rangle$ and $\left|5_{A}\right\rangle$ states to the $\left|3_{L}\right\rangle$ and $\left|3_{D}\right\rangle$ states. In this process only the $\left|4_{D}\right\rangle$ state is populated, while the $\left|3_{D}\right\rangle$ and $\left|4_{L}\right\rangle$ states become empty at the end of the process. This is because the empty pair of states is coupled to the $\left|5_{S}\right\rangle$ and $\left|5_{A}\right\rangle$ states by vectors of Rabi frequencies $\Omega_{i}$, which are orthogonal to analogous vectors of the populated pair of states, respectively. The chiral conversion of the excited enantiomer is achieved by choosing $\Omega_{4,5 S}(t)$ to have the same sign as $\Omega_{3,5 S}(t)$ and $\Omega_{4,5 A}(t)$ to have an opposite sign to $\Omega_{3,5 A}(t)$.

The conversion step in the RWA and neglecting all off-resonant terms is described by the Hamiltonian
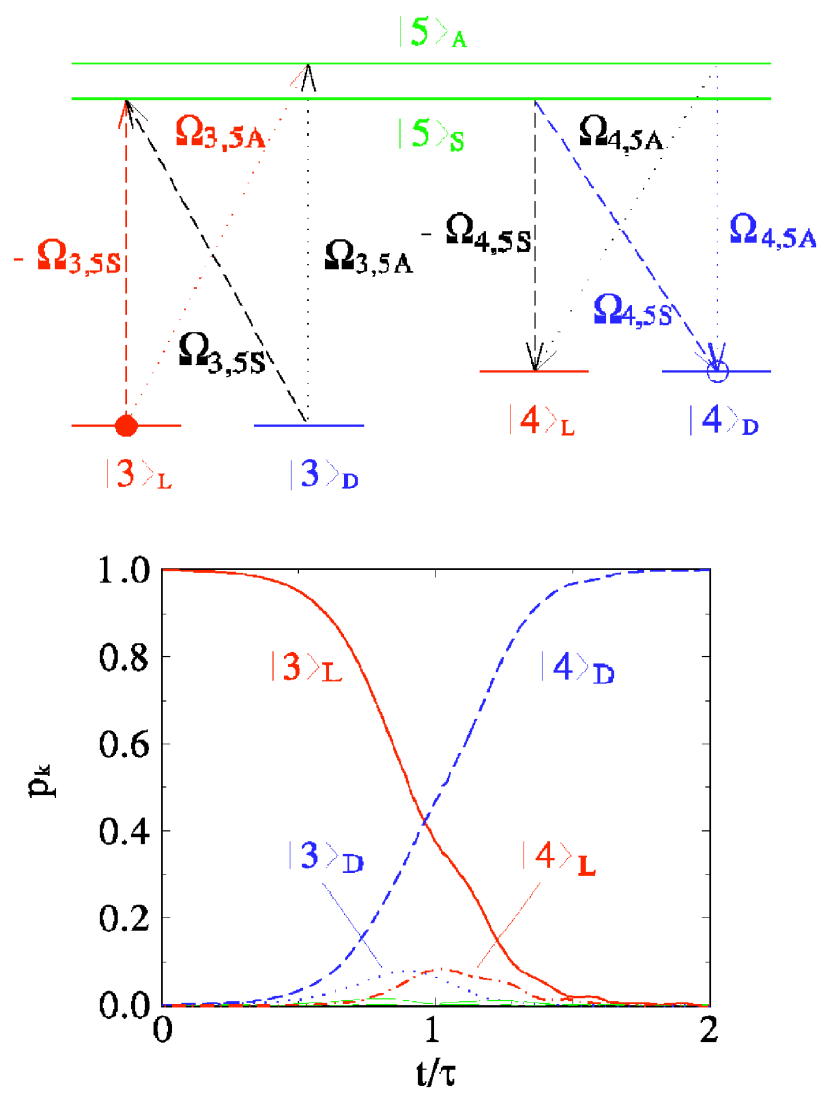

FIG. 14. (Color online) The enantioconverter scheme. (Top) Population is transferred from the $\left|3_{L}\right\rangle$ state to the $\left|4_{D}\right\rangle$ state, while going through the superposition of $\left|5_{S, A}\right\rangle$ states. (Bottom) The populations $p_{i}$ of the broken-symmetry states, as a function of time, during the conversion step.

$$
\mathrm{H}=\frac{\hbar}{2}\left[\begin{array}{cccccc}
0 & 0 & -\Omega_{3,5 S}^{*} & \Omega_{3,5 A}^{*} & 0 & 0 \\
0 & 0 & \Omega_{3,5 S}^{*} & \Omega_{3,5 A}^{*} & 0 & 0 \\
-\Omega_{3,5 S} & \Omega_{3,5 S} & 0 & 0 & -\Omega_{4,5 S}^{*} & \Omega_{4,5 S}^{*} \\
\Omega_{3,5 A} & \Omega_{3,5 A} & 0 & 0 & \Omega_{4,5 A}^{*} & \Omega_{4,5 A}^{*} \\
0 & 0 & -\Omega_{4,5 S} & \Omega_{4,5 A} & 0 & 0 \\
0 & 0 & \Omega_{4,5 S} & \Omega_{4,5 A} & 0 & 0
\end{array}\right] \text {, }
$$

with the time-dependent wave function given by $\mathbf{c}(t)$ $=\left(c_{3 L}, c_{3 D}, c_{5 S}, c_{5 A}, c_{4 L}, c_{4 D}\right)^{\mathrm{T}}$ the vector of expansion coefficients after solving Eq. (8). This Hamiltonian matrix has four nonzero eigenvalues and two null eigenvalues $\lambda_{1,2}=0$, which correspond to two dark states with coefficient vectors $\mathbf{c}_{1}(t)=\left(-d_{+},-d_{-}, 0,0,2 \Omega_{3,5 S}, 0\right)^{\mathrm{T}}$ and $\mathbf{c}_{2}(t)$ $=\left(-d_{-}, d_{+}, 0,0,0,2 \Omega_{3,5 S}\right)^{\mathrm{T}}$, where $d_{ \pm} \equiv \Omega_{4,5 S} \pm r \Omega_{4,5 A}$ and $r \equiv \Omega_{3,5 S} / \Omega_{3,5 A}$.

These expressions show that the system can follow two possible paths, of which only one results in chiral flipping of the initial state. Assuming, for simplicity, that $r=1$ and $r^{\prime}=\Omega_{4,5 S} / \Omega_{4,5 A}=1$, we find that at the beginning of the process only the null eigenvalue state $\mathbf{c}_{1}\left(t_{\text {ini }}\right)$ correlates with the initial state $\left|3_{L}\right\rangle$, i.e., the vector $\mathbf{c}\left(t_{\text {ini }}\right)$ $=(1,0,0,0,0,0)^{\mathrm{T}}$. At the end of the processes, this dark state correlates with the vector $\mathbf{c}_{1}\left(t_{\text {end }}\right)=(0,0,0,0,1,0)^{\mathrm{T}}$ 
for the $\left|4_{L}\right\rangle$ state, so the symmetry is preserved. On the other hand, if we flip the phase of just one Stokes or one pump field component $\left(r=-1\right.$ or $\left.r^{\prime}=-1\right)$, the system follows the dark state $\mathbf{c}_{2}$, which correlates at the end with the state $\mathbf{c}_{2}\left(t_{\text {end }}\right)=(0,0,0,0,0,1)^{\mathrm{T}}$. The final population thus occupies the $\left|4_{D}\right\rangle$ state, with opposite chirality, as required. We note that the transfer dynamics during the conversion step can be understood as the dynamics of two in-parallel executed three-level STIRAP processes, which is obvious when the conversion step is formulated in the $\{3 S, 3 A, 5 S, 5 A, 4 S, 4 A\}$ basis.

In Fig. 14, we show the evolution of the calculated populations $p_{i}=\left|c_{i}\right|^{2}$. The process starts in the $\left|3_{L}\right\rangle$ state and ends in the $\left|4_{D}\right\rangle$ state. The Rabi frequencies are $\Omega_{3,5 S}(t)=\Omega^{\max } f(t-2 \tau), \Omega_{3,5 A}(t)=0.5 \Omega^{\max } f(t-2 \tau), \Omega_{4,5 S}(t)$ $=0.4 \Omega^{\max } f(t)$, and $\quad \Omega_{4,5 A}(t)=-\Omega^{\max } f(t)$, with $\Omega^{\max }$ $=60 \mathrm{~ns}^{-1}$ and $f(t)$ as in the discrimination step. The transfer is complete and exclusive although, in the calculations presented in Fig. 14, we have chosen $r=2$ and $r^{\prime}$ $=-0.4$, thus demonstrating that the process is robust, as long as $r$ and $r^{\prime}$ have the right relative signs.

Recently, the schemes discussed above have also found application in biological systems for detection and automatic repair of nucleotide base-pair mutations and targeted genome manipulation (Thanopulos and Shapiro, 2005). In Appendix A, we extend the above concepts to the nonclassical light field domain and show that the use of nonclassical light in conjunction with the CCAP method allows us to prepare novel types of radiation-matter entangled states.

\section{SUMMARY}

We have discussed the merging of the coherent control and adiabatic passage methods. The combined technique, denoted CCAP, may potentially yield both selectivity between competing channels and the completeness of the population transfer to the chosen final states. As particular applications of CCAP we have shown how to prepare arbitrary wave packets made of nondegenerate or degenerate energy states. We have also discussed the use of CCAP in the encoding and decoding of quantum information and the control of broken-symmetry systems. In particular, we have demonstrated the power of the method to induce and control isomerization between local minima resulting from the Jahn-Teller effect and the optical purification of enantiomers of chiral molecules. All these results clearly demonstrate the numerous possible applications of the CCAP methodology. Experiments aimed at demonstrating these concept are currently under way (Amitay, 2006).

\section{ACKNOWLEDGMENTS}

Fruitful discussions with Paul Brumer, Einat Frishman, David Gerbasi, Bruce W. Shore, and many other researchers are gratefully acknowledged.

\section{APPENDIX A: QUANTUM-FIELD COHERENT CONTROL: PREPARATION OF ENTANGLED STATES}

In the discussion presented above, we have explored CCAP for classical electromagnetic fields, analogous to the semiclassical coherent states. In this appendix we examine the consequences of using nonclassical light (Peřina, 1984; Scully and Zubairy, 1997) in such problems, which was already considered in multimode Raman transitions (Král, 1990c). One of the possible applications of nonclassical light in the context of control, termed quantum-field coherent control (QCC), is the preparation of radiation-matter entangled states (Opatrný and Kurizki, 2001; Raimond et al., 2001; Bayer et al., 2003; Solano et al., 2003). The basic idea is that when a nonclassical light state with several dominant phase components is used in a phase-sensitive CCAP transfer, each of its components gives rise to a different dynamics and can transfer the matter population to a different final state, thereby giving rise to phasedependent light-matter entanglement (Král et al., 2005).

As an example, we discuss the extension of the onephoton versus two-photon $1+2 \mathrm{CC}$ scenario of Sec. II.A (Kurizki et al., 1989; Král and Tománek, 1999; Král and Sipe, 2000) to the case in which the one-photon pathway is executed with nonclassical light. In a one-dimensional model, where we excite the $\left|\mathbf{k}_{c}\right\rangle$ states, it is easy to show that $e^{i \delta_{1,2}}$ of Eq. (3) is simply related to the sign of $k=k_{c} \approx k_{v}$ (Král and Tománek, 1999). Hence the interference term can be written as

$$
P(k) \propto P_{\text {non }}(|k|)+P_{\text {int }}(|k|) \operatorname{sgn}(k) \cos \left(\phi_{1}-2 \phi_{2}\right) .
$$

This dependence can also be deduced from parity considerations, since the one-photon matrix element changes sign when $k$ changes sign, while the two-photon matrix does not.

In our application of the $1+2$ QCC, we use for onephoton nonclassical light a "cat state" (Yurke and Stoler, 1986; Schleich et al., 1991) of the form $|\mathcal{C}\rangle \equiv 1 / \sqrt{2}(|\alpha\rangle$ $+|-\alpha\rangle$ ), although other nonclassical states have a similar effect. This cat state is composed of two $| \pm \alpha\rangle$ coherent states whose effective phases differ by $\pi$. The probability $P\left(k_{c} ; \alpha^{\prime}\right)$ of populating the $\left|k_{c}\right\rangle$ material eigenstate and the light field in the $\left|\alpha^{\prime}\right\rangle$ coherent state can be evaluated from Eq. (3) by replacing the classical-light expression for the one-photon amplitude, $f_{1 \mathrm{phot}}=A_{1} p_{c, v} e^{-i \phi_{1}}$, with the expression appropriate for nonclassical light, $f_{1 \text { phot }}=\left\langle\alpha^{\prime}\right|\left(\Sigma_{n} p_{c v} \Sigma_{n}|n-1\rangle\langle n|+\right.$ H.c. $)|\mathcal{C}\rangle$, where $|n\rangle$ denotes Fock number states. Choosing, for simplicity, $\alpha$ $\approx \alpha^{\prime}$ to be real and using the fact (Peřina, 1984) that $\left\langle n \mid \alpha^{\prime}\right\rangle \propto\left(\alpha^{\prime}\right)^{n} / \sqrt{n}$, we can write $P\left(k_{c} ; \alpha^{\prime}\right)$ as

$$
P\left(k ; \alpha^{\prime}\right) \propto P_{\text {non }}(|k|)+P_{\text {int }}(|k|) \operatorname{sgn}\left(\alpha^{\prime}\right) \operatorname{sgn}(k) \cos \left(2 \phi_{2}\right) .
$$

The additional $\operatorname{sgn}\left(\alpha^{\prime}\right)$ term derives from the relation $\left\langle\alpha^{\prime} \mid n-1\right\rangle\langle n \mid \mathcal{C}\rangle=\left[1+(-1)^{n}\right]\left(\alpha^{\prime}\right)^{n-1} \alpha^{n} / \sqrt{2 n(n-1)}$. We see that only even $n$ (odd $n-1$ ) terms contribute to the sum, and all these terms have the same sign as $\alpha^{\prime}$. 


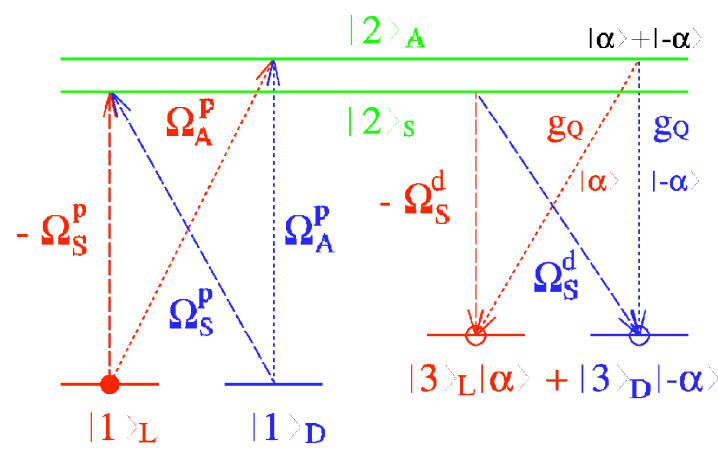

FIG. 15. (Color online) The nonclassical enantioconverter. In the classical-field converter, an initial (left-handed) $\left|1_{L}\right\rangle$ state is transformed into the $\left|3_{D}\right\rangle$ state. The nonclassical converter uses the $|\alpha\rangle+|-\alpha\rangle$ cat state to produce the $\left|3_{L}\right\rangle|\alpha\rangle+\left|3_{D}\right\rangle|-\alpha\rangle$ entangled state.

The apparently small difference between Eqs. (3) and (A2), i.e., the correlation of the signs in $\alpha^{\prime}$ and $k$, has far reaching consequences. By setting the fields such that the magnitudes of the interference term and the noninterfering sum be the same, $P_{\text {non }}(|k|)=P_{\text {int }}(|k|)$, and choosing for simplicity $\phi_{2}=0$, we obtain that $P\left(k ; \alpha^{\prime}\right)$ $=0$ if $\operatorname{sgn}(\alpha) \neq \operatorname{sgn}(k)$. Therefore, by starting from the initial state of the occupied valence band, $\rho_{v v}^{0}=\Sigma_{k_{v}}\left|k_{v}\right\rangle\left\langle k_{v}\right|$, we can prepare a mixture of the left and right momenta $\left|-k_{c, v}\right\rangle$ and $\left|k_{c, v}\right\rangle$ states (currents) correlated with the $|-\alpha\rangle$ and $|\alpha\rangle$ coherent states, respectively. If we start from a pure state of a quantum well, this approach could prepare entangled states of the form $|\Psi\rangle \approx\left|k_{c}\right\rangle|\alpha\rangle$ $+\left|-k_{c}\right\rangle|-\alpha\rangle$. Since the $1+2 \mathrm{CC}$ can also inject spin currents in semiconductors (Bhat and Sipe, 2000), the QCC could prepare entangled spin currents.

The presented example demonstrates the QCC in the weak-field (perturbative) limit, where broken-symmetry material states in a continuum are entangled with nonclassical radiation states. As mentioned in the Introduction, this is the regime where the traditional CC is applied, although both CC and QCC can work with strong fields as well. We now extend the QCC approach to the strong-field (nonperturbative) limit in such a way that it will induce complete transitions, as outlined in the CCAP approach, between discrete broken-symmetry material states. In the quantum-field domain, use of AP methods has been suggested as a way of preparing Focknumber states (Parkins et al., 1993, 1995; Hennrich et al., 2000; Gong et al., 2002) and certain entangled material states (Unanyan, Shore, and Bergmann, 2001; Unanyan, Vitanov, and Bergmann, 2001; Unanyan et al., 2002;).

Here, as an example of strong-field QCC, we analyze the use of nonclassical fields in preparing radiationmatter chiral entangled states (Král et al., 2005). We extend the treatment of chiral purification of Sec. VI to the enantioconversion process. As explained there, this process is used to transfer populations from an initial lefthanded state, say $\left|1_{L}\right\rangle$, to a right-handed state, say $\left|3_{D}\right\rangle$, using delocalized states, say $\left|2_{S}\right\rangle$ and $\left|2_{A}\right\rangle$, as intermediates. The entanglement in the converter can be realized by replacing one of the four light fields by the nonclas- sical $|\mathcal{C}\rangle$ cat state. Since the two coherent $| \pm \alpha\rangle$ components of opposite quantum phases drive the system along the two mirror-imaged paths (see Fig. 15), the quantum-field enantioconverter populates the entangled radiation-matter $|\Psi\rangle=\left(\left|3_{L}\right\rangle|\alpha\rangle+\left|3_{D}\right\rangle|-\alpha\rangle\right) / \sqrt{2}$ state.

The situation can be simulated quantitatively by expanding the system wave function as

$$
|\Psi(t)\rangle=\sum_{k=1}^{6} \sum_{n=0}^{\infty} c_{k, n}(t) e^{-i\left(\omega_{k}+n \omega_{0}\right) t}|k, n\rangle,
$$

where $k=\{1 L, 1 D, 2 S, 2 A, 3 L, 3 D\}$ are the molecular states and $n$ denotes the number of photons in the nonclassical Stokes field of frequency $\omega_{0}$, which can be obtained by solving the Schrödinger equation:

$$
\begin{aligned}
i \dot{c}_{1 L, n}= & \left(\Omega_{S}^{p} / 2\right) c_{2 S, n}+\left(\Omega_{A}^{p} / 2\right) c_{2 A, n}, \\
i \dot{c}_{1 D, n}= & \left(\Omega_{S}^{p} / 2\right) c_{2 S, n}-\left(\Omega_{A}^{p} / 2\right) c_{2 A, n}, \\
i \dot{c}_{2 S, n}= & \left(\Omega_{S}^{p^{*}} / 2\right)\left(c_{1 L, n}+c_{1 D, n}\right)+\left(\Omega_{S}^{d^{*}} / 2\right)\left(c_{3 L, n}+c_{3 D, n}\right), \\
i \dot{c}_{2 A, n}= & \left(\Omega_{A}^{p^{*}} / 2\right)\left(c_{1 L, n}-c_{1 D, n}\right)+\left(g_{Q} / 2\right) \sqrt{n+1}\left(c_{3 L, n+1}\right. \\
& \left.-c_{3 D, n+1}\right), \\
i \dot{c}_{3 L, n}= & \left(\Omega_{S}^{d} / 2\right) c_{2 S, n}+\left(g_{Q} / 2\right) \sqrt{n} c_{2 A, n-1}, \\
i \dot{c}_{3 D, n}= & \left(\Omega_{S}^{d} / 2\right) c_{2 S, n}-\left(g_{Q} / 2\right) \sqrt{n} c_{2 A, n-1} .
\end{aligned}
$$

From the numerical solution of $|\Psi(t)\rangle$, we can obtain the density operator $\rho(t)=|\Psi(t)\rangle\langle\Psi(t)|$. The system entanglement can be examined from its diagonal matrix elements $\rho_{k, k}^{\beta, \beta}(t) \equiv\langle k, \beta|\rho(t)| k, \beta\rangle(k=1-6)$ in the (overcomplete) coherent-state $|\beta\rangle$ basis (Peřina, 1984), evaluated in terms of the Fock-state matrix elements as

$$
\rho_{k, k}^{\beta, \beta}(t)=\sum_{n=0}^{\infty} \sum_{m=0}^{\infty}\langle\beta \mid n\rangle c_{k}^{n}(t)\left(c_{k}^{m}(t)\right)^{*}\langle m \mid \beta\rangle .
$$

We can plot the elements as a function of the real and imaginary parts of $\beta$, i.e., $\beta_{x}=\operatorname{Re} \beta$ and $\beta_{y}=\operatorname{Im} \beta$.

In Fig. 16, we display the final-state matrix elements $\rho_{L, L}^{\beta, \beta}$ and $\rho_{D, D}^{\beta, \beta}$ obtained by solving Eq. (A4) for the Rabi frequencies $\Omega_{S}^{p}(t)=\Omega_{A}^{p}(t)=\Omega_{0} f(t-2 \tau), \Omega_{S}^{d}(t)=\Omega_{0} f(t)$, and $g_{Q}(t)=g_{0} f(t)$, with $f(t)=\exp \left[-t^{2} / \tau^{2}\right], \tau=30, \Omega_{0}=2$, and $g_{0}$ $=0.2$. The left column shows the results of driving the system with the $|\mathcal{C}\rangle$ cat state, with $|\alpha|^{2}=7$. Starting from the $\left|1_{L}\right\rangle$ state, we prepare the entangled $|\Psi\rangle \approx\left|3_{L}\right\rangle|\alpha\rangle$ $+\left|3_{D}\right\rangle|-\alpha\rangle$ state.

In Fig. 16 (right column), we show the results of driving the system using the $|n=7\rangle$ Fock state, where we obtain the final entangled state

$$
\begin{aligned}
|\Psi\rangle & \approx\left|3_{L}\right\rangle(|n+1\rangle+|n\rangle)+\left|3_{D}\right\rangle(|n+1\rangle-|n\rangle) \\
& \approx\left|3_{S}\right\rangle|n+1\rangle+\left|3_{A}\right\rangle|n\rangle .
\end{aligned}
$$

We can see the formation of the $|n=8\rangle_{ \pm}|n=7\rangle$ states. These states with "bananalike" distributions are related to the multiphase $\Sigma_{n}\left|e^{i 2 \pi / n} \alpha\right\rangle$ coherent states generated by the nonlinear Kerr effect (Král, 1990b). 


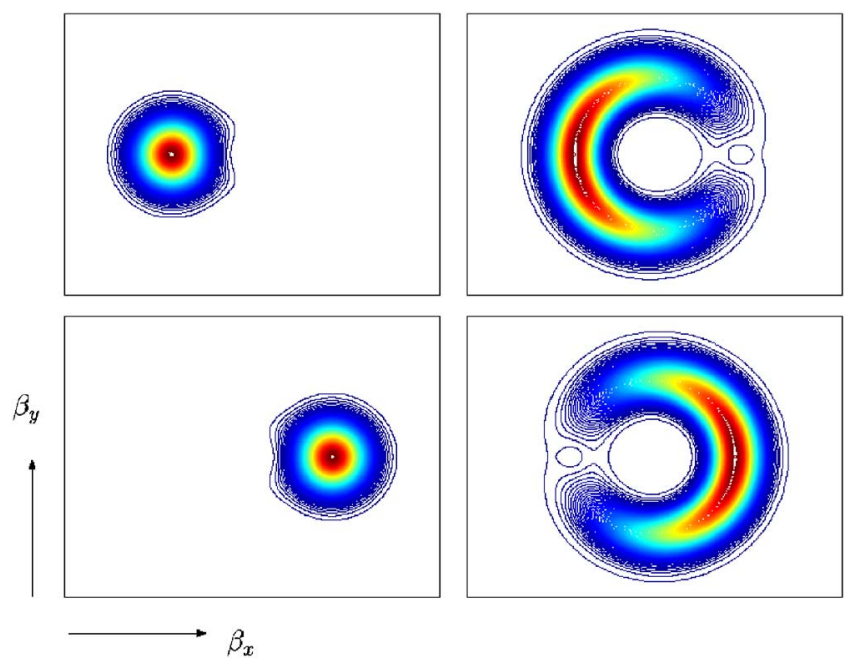

FIG. 16. (Color online) Photon distributions $\rho_{L, L}^{\beta, \beta}$ (upper panels) and $\rho_{D, D}^{\beta, \beta}$ (lower panels) in the quantum field enantioconverter for entangled chiral states. The distributions are presented in the $\Delta \beta_{x}= \pm 6.4, \Delta \beta_{y}= \pm 5$ range. (Left column) The driving field is given by the cat state $|\mathcal{C}\rangle$ with $|\alpha|^{2}=7$. (Right column) The driving field is given by the $|n=7\rangle$ Fock state.

\section{APPENDIX B: DERIVATION OF THE TRANSPORT EQUATIONS FOR POLYCHROMATIC PASSAGE SCHEMES}

In this appendix, we first formally obtain the Schrödinger equation (8) in the interaction picture. This can be done by transforming the Schrödinger-picture equation (6) with the unitary transformation $U_{0}(t)$ $=\exp \left(-i H_{M} t / \hbar\right)\left[U_{0}^{\dagger}(t) U_{0}(t)=1\right]$ (Shore, 1990; Scully and Zubairy, 1997) as follows:

$$
\begin{aligned}
& U_{0}^{\dagger}(t) \frac{\partial}{\partial t}|\Psi(t)\rangle=-\frac{i}{\hbar} U_{0}^{\dagger}(t) H(t) U_{0}(t) U_{0}^{\dagger}(t)|\Psi(t)\rangle, \\
& \frac{\partial}{\partial t}|\tilde{\Psi}(t)\rangle-\left[\frac{\partial}{\partial t} U_{0}^{\dagger}(t)\right]|\Psi(t)\rangle=-\frac{i}{\hbar}\left[H_{M}+\mathrm{H}(t)\right]|\tilde{\Psi}(t)\rangle, \\
& \frac{\partial}{\partial t}|\tilde{\Psi}(t)\rangle=-\frac{i}{\hbar} \mathrm{H}(t)|\tilde{\Psi}(t)\rangle .
\end{aligned}
$$

Here, we have used the fact that

$$
\begin{aligned}
& U_{0}^{\dagger}(t) H(t) U_{0}(t)=H_{M}+U_{0}^{\dagger}(t) H_{I}(t) U_{0}(t)=H_{M}+\mathrm{H}(t), \\
& U_{0}^{\dagger}(t)|\Psi(t)\rangle=|\tilde{\Psi}(t)\rangle=\sum_{i=1,0,2} c_{i}(t)|i\rangle,
\end{aligned}
$$

where $c_{i}(t)=C_{i}(t) e^{i \omega_{i} t}$ and the interaction-picture wave function $|\tilde{\Psi}(t)\rangle$ is written for $|\Psi(t)\rangle$ in Eq. (7). If we write the Schrödinger equation (B1) in the interaction picture in matrix form with the bare-state basis set $|i\rangle(i$ $=1,0,2)$ and use the RWA approximation for the interaction-picture Hamiltonian $\mathrm{H}(t)$, we obtain Eq. (8).

We now generalize this description to the situation of a polychromatic excitation in multilevel systems. We consider the adiabatic passage in a material system composed of one initial, one intermediate, and many final states (Král et al., 2002). Such a system contains the essence of the NQC scheme presented in Sec. III.

We consider the action of a shaped Stokes pulse, resulting from the contributions of many pulses of different center frequencies, each resonantly coupling one of the $|k\rangle(k=3, \ldots, n+2)$ final states to a single intermediate state $|2\rangle$. This pulse precedes (while partially overlapping) the pump pulse, which couples states $|2\rangle$ and $|1\rangle$.

This situation is described by the Hamiltonian

$$
\begin{aligned}
H(t)= & H_{M}+E_{P}(t)\left[\mu_{2,1}|2\rangle\langle 1|+\text { H.c. }\right] \\
& +E_{S}(t) \sum_{k=3}^{n+2}\left[\mu_{k, 2}|k\rangle\langle 2|+\text { H.c. }\right],
\end{aligned}
$$

where we assume for simplicity only one (linear) polarization direction for the pump and Stokes electric fields $E_{P}(t)=\operatorname{Re} \mathcal{E}_{1,2}(t) e^{-i \omega_{1,2} t}$ and $E_{S}(t)=\operatorname{Re} \sum_{k=3}^{n+2} \mathcal{E}_{2, k}(t) e^{-i \omega_{2, k} t}$, respectively. The slow field amplitudes $\mathcal{E}_{i, j}(t)$ of the Stokes pulse are assumed to have the same smooth (Gaussian) time profile, characterized by a common pulse width $\tau_{\text {pulse. }}$ The frequencies $\omega_{i, j}$ of the $E_{P}(t)$ and $E_{S}(t)$ fields are chosen to be in near resonance $\left(\omega_{i, j} \approx \omega_{i}-\omega_{j}\right)$ with the relevant transition frequencies, namely, $\omega_{1,2}$ for $E_{P}$ and $\omega_{2, k}$ for $E_{S}$. As in the main text, the coupling is mediated by the dipole matrix elements $\mu_{i, j}$.

We start from the $|\Psi(t)\rangle=\sum_{i=1}^{n+2} c_{i}(t) e^{-i \omega_{i} t}|i\rangle$ wave function as in Eq. (7). The vector of $c_{i}(t)(i=1, \ldots, n+2)$ coefficients, $\mathbf{c}(t)=\left(c_{1}, c_{2}, c_{3}, \ldots, c_{n+2}\right)^{\mathrm{T}}$, is a solution of the matrix Schrödinger equation $(8), \hbar \dot{\mathbf{c}}(t)=-i \mathcal{H}(t) \cdot \mathbf{c}(t)$, with the Hamiltonian $\left(\delta_{i, j} \equiv \omega_{j}-\omega_{i}\right)$

$$
\mathcal{H}(t)=\left[\begin{array}{ccccc}
0 & E_{P} \mu_{1,2} e^{-i \delta_{1,2} t} & 0 & \cdots & 0 \\
E_{P} \mu_{2,1} e^{i \delta_{1,2} t} & 0 & E_{S} \mu_{2,3} e^{-i \delta_{2,3} t} & \cdots & E_{S} \mu_{2, n+2} e^{i \delta_{2, n+2} t} \\
0 & E_{S} \mu_{3,2} e^{i \delta_{2,3} t} & 0 & \cdots & 0 \\
\cdots & \cdots & \cdots & \cdots & \cdots \\
0 & E_{S} \mu_{n+2,2} e^{i \delta_{2, n+2} t} & 0 & \cdots & 0
\end{array}\right]
$$

If we introduce the RWA in $\mathcal{H}(t)$ for the on-resonance transitions for both pulses, as described in Sec. II.B, but retain all other components of the Stokes electric field, we obtain the RWA Hamiltonian 


$$
\mathrm{H}(t)=\frac{\hbar}{2}\left[\begin{array}{ccccc}
0 & \Omega_{1,2}(t) & 0 & \cdots & 0 \\
\Omega_{2,1}(t) & 0 & \Sigma_{2,3}(t) & \cdots & \Sigma_{2, n+2}(t) \\
0 & \Sigma_{3,2}(t) & 0 & \cdots & 0 \\
\cdots & \cdots & \cdots & \cdots & \cdots \\
0 & \Sigma_{n+2,2}(t) & 0 & \cdots & 0
\end{array}\right],
$$

where $\Omega_{i, j}(t) \equiv \mu_{i, j} \mathcal{E}_{i, j}(t) / \hbar$ are the usual (we assume real $\mu_{i, j}$ for simplicity) and $\Sigma_{2, k}(t)$ are the modified (Stokes) Rabi frequencies

$$
\Sigma_{2, k}(t)=\mu_{2, k}\left(\mathcal{E}_{2, k}(t)+\sum_{l=3 \neq k}^{n+2} e^{-i\left(\delta_{2, k^{ \pm}} \pm \omega_{2, l}\right) t} \mathcal{E}_{2, l}(t)\right)=\Omega_{2, k}(t)+\text { (off-resonant terms). }
$$

The $\Sigma_{2, k}$ elements incorporate the effects of both the on-resonance $\mathcal{E}_{2, k} \quad\left(\delta_{2, k}+\omega_{2, k} \approx 0\right)$ and off-resonance $\mathcal{E}_{2, l \neq k} \quad\left(\delta_{2, k}+\omega_{2, l} \approx \omega_{k}-\omega_{l} \quad\right.$ and $\left.\quad \delta_{2, k}-\omega_{2, l} \approx \omega_{k}+\omega_{l}-2 \omega_{2}\right)$ field components on the given $|2\rangle \rightarrow|k\rangle$ transition. We can directly integrate these equations numerically (Král et al., 2002) and check the importance of the various terms in Eq. (B6). If the field components $\mathcal{E}_{i, j}(t)$ are relatively weak so that $\left|\Omega_{i j}(t)\right| \ll\left|\omega_{i}-\omega_{i \pm 1}\right|$ the contributions of the off-resonance oscillatory terms of Eq. (B6) should be negligible, causing $\Sigma_{2, k}(t)$ to coincide with the resonant term $\Omega_{2, k}(t)$.

If we neglect the nonresonant terms in Eq. (B6), the adiabatic states can be used in the description of this problem (Shore, 1990; Scully and Zubairy, 1997). Of the $n+2$ eigenvalues of $\mathrm{H}(t), n$ are zero and two are nonzero,

$$
\begin{aligned}
& \lambda_{1,2, \ldots, n}=0, \\
& \lambda_{n+1, n+2}(t)= \pm\left(\left|\Omega_{1,2}(t)\right|^{2}+\sum_{k=3}^{n+2}\left|\Sigma_{2, k}(t)\right|^{2}\right)^{1 / 2} .
\end{aligned}
$$

The zero eigenvalues correspond to the $n$ dark states,

$$
\left|D_{k-2}(t)\right\rangle=\Sigma_{2, k}(t)|1\rangle-\Omega_{2,1}(t)|k\rangle \quad(k=3, \ldots, n+2),
$$

mixing the initial $|1\rangle$ and final $|k\rangle$ states. In Appendix C, we show how such dark states can be combined to form a single "transferring" state and many "nontransferring" states orthogonal and uncoupled to it during the process, thus rendering the evolution of the transferring state fully adiabatic in this case, as well as in the general NQC case of many initial and final nondegenerate states.

\section{APPENDIX C: ADIABATIC EVOLUTION IN POLYCHROMATIC PASSAGE SCHEMES}

In this appendix, we discuss first the general conditions for adiabatic population transfer, using the system presented in Appendix B. We then show the particular conditions valid for the schemes discussed in this work, which allow for such a transfer even in case of systems with many dark states.

When, besides the (off-resonance) conditions discussed below Eq. (B6), the following conditions are sat- isfied, we expect the adiabatic solution for the time evolution of the system, based on diagonalizing the RWA Hamiltonian $\mathrm{H}(t)$ in Eq. (B5), to be accurate. The first one is the global adiabaticity condition of large pulse area, $1 /\left|\max \left\{\mathcal{E}_{i}(t)\right\}\right| \ll \tau_{\text {pulse }}$, which can, in principle, be always satisfied if all fields are strong enough. The second condition of the local adiabaticity [for an in-depth discussion of adiabatic conditions see Shore (1990) and Vitanov et al. (2001)], $\left(\mathrm{U}^{-1}(t) \cdot \dot{\mathrm{U}}(t)\right)_{k, l} \ll\left|\lambda_{k}(t)-\lambda_{l}(t)\right|$, might be satisfied when the field components $\mathcal{E}_{i, j}(t)$ in Eq. (B6) change slowly enough with time. Here $\mathrm{U}(t)$ is the matrix of eigenvectors and $\lambda_{k}(t)$ are the eigenstates of the system in Eq. (B7). This condition is violated when degenerate eigenstates are present in the spectrum of the RWA Hamiltonian, such as in Eq. (B7).

Let us now explain why this degeneracy problem does not arise in the schemes presented in Secs. III and V, which have multiple dark states. This fact is due to zero nonadiabatic couplings between the dark eigenstates in these systems for the chosen pulses, shown below analytically for the general NQC scheme discussed in Sec. III. This proof was demonstrated numerically for a fourlevel polychromatic scheme analogous to that presented in Appendix B, as given by Král and Shapiro (2002).

We first consider the INS and FNS states of Sec. III defined in Eq. (17). From their definition, we have that the single transferring MNS state of Eq. (18), $\left|D^{M}(t)\right\rangle$ $\propto \Sigma_{k} c_{k}^{i}(t)|k\rangle-\sum_{l} c_{l}^{f}(t)|l\rangle$, is orthogonal to the INS and FNS states at all times. This is because the time-dependent (normalized) vectors $\mathbf{c}^{i}(t)=\left(c_{1}^{i}, c_{2}^{i}, \ldots, c_{n}^{i}\right)^{\mathrm{T}}=\cos \theta(t) \mathbf{c}_{0}^{i}$ and $\mathbf{c}^{f}(t)=\left(c_{1}^{f}, c_{2}^{f}, \ldots, c_{m}^{f}\right)^{\mathrm{T}}=\sin \theta(t) \mathbf{c}_{0}^{f} \quad$ fulfill $\quad\left(\mathbf{d}^{i}(t)\right)^{\dagger} \cdot \mathbf{c}^{i}(t)$ $=\left(\mathbf{d}_{0}^{i}\right)^{\dagger} \cdot \mathbf{c}_{0}^{i} \cos \theta(t)=0$ and $\left(\mathbf{d}^{f}(t)\right)^{\dagger} \cdot \mathbf{c}^{f}(t)=\left(\mathbf{d}_{0}^{f}\right)^{\dagger} \cdot \mathbf{c}_{0}^{f} \sin \theta(t)$ $=0$, since $\mathbf{c}_{0}^{i}$ and $\mathbf{c}_{0}^{\mathbf{f}}$ are chosen in such a way (see also Sec. III) that $\left(\mathbf{d}_{0}^{i}\right)^{\dagger} \cdot \mathbf{c}_{0}^{i}=0$ and $\left(\mathbf{d}_{0}^{f}\right)^{\dagger} \cdot \mathbf{c}_{0}^{f}=0$, where $\mathbf{d}_{0}^{i}$ $=\mathbf{d}^{i}\left(t_{\text {in }}\right)$ and $\mathbf{d}_{0}^{f}=\mathbf{d}^{f}\left(t_{\text {end }}\right)$ are the (normalized) vectors with the coefficients of the individual INS and FNS states in Eq. (17), respectively. All MNS, INS, and FNS states are also orthogonal to the two bright states of the system.

We can now transform the system to a new orthogonal basis in the eigenvector space that is formed by the single transferring MNS state by $n+m-2$ dark states formed by $n-1$ linearly independent combinations of 
the INS states and $m-1$ combinations of the FNS states, as well as by two bright states. The Schrödinger equation (8) transformed to the space of these new eigenstates (Kobrak and Rice, 1998a; Unanyan, Fleischhauer, Shore, and Bergmann, 1998; Kis and Stenholm, 2001; Král and Shapiro, 2002) looks as follows: $\hbar \dot{\mathbf{v}}(t)$ $=-i \mathbf{W}(t) \cdot \mathbf{v}(t)$, where $\mathbf{v}(t)=\mathbf{U}(t) \cdot \mathbf{c}(t)$ is the new state vector. The matrix $\mathbf{U}(t)$ of eigenvectors diagonalizes the RWA Hamiltonian $\mathrm{H}(t)$ and generates the evolution ma$\operatorname{trix} \mathbf{W}(t) \equiv \mathbf{U}^{-1}(t) \cdot \mathrm{H}(t) \cdot \mathbf{U}(t)-i \hbar \mathbf{U}^{-1}(t) \cdot \dot{\mathbf{U}}(t)$. The first term in $\mathbf{W}(t)$ is diagonal, and its dark-space part, on which we can limit our study, is zero. The second term describes the crucial nonadiabatic coupling between dark states.

We now calculate the nonadiabatic coupling matrix elements in the dark eigenvector subspace. Since the Hamiltonian is Hermitian, the matrix of its eigenstates fulfills $\mathbf{U}^{-1}(t)=\left(\mathbf{U}^{\mathrm{T}}\right)^{*}(t) \equiv \mathbf{U}^{\dagger}(t)$. We use this equality in calculating the scalar products between the terms in the $\mathbf{U}^{-1}(t) \cdot \dot{\mathbf{U}}(t)$ matrix. The time derivative of the vector of coefficients for the MNS state, $\mathbf{c}(t) \propto \mathbf{c}^{i}(t)-\mathbf{c}^{f}(t)$ $=\cos \theta(t) \mathbf{c}_{0}^{i}-\sin \theta(t) \mathbf{c}_{0}^{f}$, has the form $\dot{\mathbf{c}}(t) \propto-\dot{\theta}(t) \sin \theta(t) \mathbf{c}_{0}^{i}$ $-\dot{\theta}(t) \cos \theta(t) \mathbf{c}_{0}^{f}$. Therefore, we obtain that $\left(\mathbf{d}^{i}(t)\right)^{\dagger} \cdot \dot{\mathbf{c}}(t)$ $=-\left(\mathbf{d}_{0}^{i}\right)^{\dagger} \cdot \mathbf{c}_{0}^{i} \dot{\theta}(t) \sin \theta(t)=0 \quad$ and $\quad\left(\mathbf{d}^{f}(t)\right)^{\dagger} \cdot \dot{\mathbf{c}}(t)=\left(\mathbf{d}_{0}^{f}\right)^{\dagger} \cdot \mathbf{c}_{0}^{f} \dot{\theta}(t)$ $\times \cos \theta(t)=0$. There is thus no coupling between the transferring MNS state and the INS and FNS dark states and, of course, linear combinations of them. The coupling terms including time derivatives of the INS and FNS dark states are also vanishing, since as follows from the definition of Eq. (17) the time derivative of any INS and FNS state is zero. Therefore, the off-diagonal ele-

ments of the $\mathbf{U}^{-1}(t) \cdot \dot{\mathbf{U}}(t)$ matrix in the dark subspace are all zero. This proves that the population follows adiabatically the transferring state during the process. One could analogously show that the $\mathbf{U}^{-1}(t) \cdot \dot{\mathbf{U}}(t)$ matrix in the dark subspace of the scheme presented in Sec. $\mathrm{V}$ is also zero.

\section{APPENDIX D: SELECTION RULES IN OPTICAL TRANSITIONS}

The scheme in Appendix B can be applied, for example, to the transfer of populations between vibronic states of the $\mathrm{Na}_{2}$ molecule, where the initial and final states belong to the ground electronic surface $X^{1} \Sigma_{g}^{+}$and the intermediate state to the higher electronic surface $A^{1} \Sigma_{u}^{+}$. Following the Franck-Condon approximation (Franck, 1925; Condon, 1928; Herzberg, 1950), according to which the electric-dipole transition matrix elements can be approximated as $\left\langle A^{1} \Sigma_{u}^{+}, v^{\prime}=0|\hat{\epsilon} \cdot \vec{\mu}| X^{1} \Sigma_{g}^{+}, v\right\rangle$ $\approx \bar{\mu}\langle 0 \mid v\rangle$, a broad progression of transitions is expected due to the large shift in the equilibrium distances between different electronic states of $\mathrm{Na}_{2}$. It is thus possible to prepare a final wave packet composed of many individual eigenstates on the ground potential surface.

When parity is well defined, such as in the $\mathrm{Na}_{2}$ molecule, electric-dipole absorption and emission of one photon entail a change of parities and those of two photons maintain the parity of the initial and final material states. In broken-symmetry systems, such as in the chiral $\mathrm{D}_{2} \mathrm{~S}_{2}$ molecule, there is no center of inversion and the states are of ill-defined parity. Thus, for such (chiral) systems the above selection rules are broken. Although the states with well-defined parity are the true eigenstates of the system, in chiral systems where the barrier for the interconversion between enantiomers is very high, the eigenstates are never observed.

In order to calculate the transition dipole matrix elements $\vec{\mu}_{1,2} \equiv\left\langle\Psi_{1}(\mathbf{q})|\vec{\mu}(\mathbf{q})| \Psi_{2}(\mathbf{q})\right\rangle$ between the $\Psi_{i}(\mathbf{q})$ $(i=1,2)$ broken-symmetry states, we can express each such states as a linear combination of rovibrational eigenstates of well-defined parities. The rovibrational eigenstates are in turn obtained by solving the corresponding rovibrational Schrödinger equation [see, e.g., Marquardt et al. $(2000,2003)$ and Thanopulos et al. (2003)].

In solid-state materials, such as in $\mathrm{Si}$ or GaAs bulk semiconductors, the selection rules are dictated by the symmetry of the crystal. The dipole elements can be evaluated by first-principle techniques (Král and Sipe, 2000). Away from the center of the Brillouine zone, the states have neither even nor odd symmetry. Nevertheless, the strength of the $1+2 \mathrm{CC}$ excitation process can be shown to depend on the $\operatorname{sgn}(\mathbf{k})$ factor. Such light excitation thus breaks the symmetry of the material, which leads to the photogeneration of electric currents.

\section{REFERENCES}

Agarwal, G. S., and W. Harshawardhan, 1996, Phys. Rev. Lett. 77, 1039.

Amitay, Z., 2006, unpublished.

Atanasov, R., A. Haché, L. P. Hughes, H. M. van Driel, and J.

E. Sipe, 1996, Phys. Rev. Lett. 76, 1703.

Aubanel, E. E., and A. D. Bandrauk, 1997, Can. J. Chem. 107, 1441.

Band, Y. B., and P. S. Julienne, 1991, J. Chem. Phys. 94, 5291. Band, Y. B., and P. S. Julienne, 1992, J. Chem. Phys. 96, 3339. Baranova, B., A. Chudinov, and B. Y. Zel'dovitch, 1990, Opt. Commun. 79, 116.

Bayer, M., P. Hawrylak, K. Hinzer, S. Fafard, M. K. Z. R. Wasilewski, O. Stern, and A. Forchel, 2003, Science 291, 451. Bergmann, K., and W. Demtröder, 1971, Z. Phys. 243, 1.

Bergmann, K., H. Theuer, and B. W. Shore, 1998, Rev. Mod. Phys. 70, 1003.

Berman, P. R., 1998, Phys. Rev. A 58, 4886.

Berry, M. V., 1984, Proc. R. Soc. London, Ser. A 392, 45.

Beumee, J. G. B., and H. Rabitz, 1990, J. Math. Phys. 31, 1253. Bhat, R. D. R., and J. Sipe, 2000, Phys. Rev. Lett. 85, 5432.

Bodenhöfer, K., A. Hiermann, J. Seemann, G. G. B. Koppenhoefer, and W. Göpel, 1997, Nature (London) 387, 577.

Boldyrev, A. I., and P. von R. Schleyer, 1991, J. Am. Chem. Soc. 113, 9045 .

Borst, D., and D. Pratt, 2000, J. Chem. Phys. 113, 3658.

Braunstein, S. L., and H. J. Kimble, 2000, Phys. Rev. A 61, 042302.

Brumer, P., and M. Shapiro, 1986, Chem. Phys. Lett. 126, 541. 
Bunker, P. R., and P. Jensen, 1998, Molecular Symmetry and Spectroscopy (NRC Research Press, Ottawa).

Carroll, C. E., and F. T. Hioe, 1995, Phys. Lett. A 199, 145.

Carroll, C. E., and F. T. Hioe, 1996, Phys. Rev. A 54, 5147.

Chen, C., Y.-Y. Yin, and D. Elliot, 1990, Phys. Rev. Lett. 64, 507.

Condon, E. U., 1928, Phys. Rev. 32, 858.

Coulston, G. W., and K. Bergmann, 1992, J. Chem. Phys. 96, 3467.

Crim, F. F., 1984, Annu. Rev. Phys. Chem. 35, 657.

Crim, F. F., 1993, Annu. Rev. Phys. Chem. 44, 397.

Dayan, N. D. B., S. G. Faeder, and Y. Silberberg, 2001, Phys. Rev. Lett. 86, 47.

Dayan, B., A. Pe'er, A. A. Friesem, and Y. Silberberg, 2004, Phys. Rev. Lett. 93, 023005.

de Araujo, L. E. E., 1999, J. Phys. Chem. A 103, 10409.

Deyirmenjian, V. B., and J. E. Sipe, 1999, Phys. Rev. Lett. 82, 4942.

Duan, L.-M., M. D. Lukin, J. I. Cirac, and P. Zoller, 2001, Nature (London) 414, 413.

Dumke, R., J. D. Weinstein, M. Johanning, K. Jones, and P. Lett, 2005, Phys. Rev. A 72, 041801.

Dupont, E., P. B. Corkum, H. C. Liu, M. Buchanan, and Z. R. Wasilewski, 1995, Phys. Rev. Lett. 74, 3596.

Esslinger, T., F. Sander, M. Weidemüller, A. Hammerich, and T. W. Hänsch, 1996, Phys. Rev. Lett. 76, 2432.

Fijimura, Y., L. Gonzalez, K. Hoki, J. Manz, and Y. Ohtsuki, 1999, Chem. Phys. Lett. 306, 1.

Fleischhauer, M., and A. S. Manka, 1996, Phys. Rev. A 54, 794.

Fleischhauer, M., R. Unanyan, B. W. Shore, and K. Bergmann, 1999, Phys. Rev. A 59, 3751.

Franck, J., 1925, Trans. Faraday Soc. 21, 536.

Frisch, M. J., et al., 2001, computer code GAUSSIAN 98, revision A.11, Gaussian, Inc., Pittsburgh PA.

Frishman, E., and M. Shapiro, 2001, Phys. Rev. Lett. 87, 253001.

Frishman, E., and M. Shapiro, 2003, Phys. Rev. A 68, 032717.

Frishman, E., M. Shapiro, D. Gerbasi, and P. Brumer, 2003, J. Chem. Phys. 119, 7237.

Gao, J.-Y., S.-H. Yang, D. Wang, X.-Z. Guo, K.-X. Chen, Y. Jiang, and B. Zhao, 2000, Phys. Rev. A 61, 023401.

Garraway, B. M., and K.-A. Suominen, 1998, Phys. Rev. Lett. 80, 932.

Gaubatz, U., P. Rudecki, M. Becker, S. Schiemann, M. Külz, and K. Bergmann, 1988, Chem. Phys. Lett. 149, 463.

Gaubatz, U., P. Rudecki, S. Sciemann, and K. Bergmann, 1990, J. Chem. Phys. 92, 5363.

Gerbasi, D., M. Shapiro, and P. Brumer, 2001, J. Chem. Phys. 115, 5349 .

Glushko, B., and B. Kryzhanovsky, 1992, Phys. Rev. A 46, 2823.

Gong, S.-Q., R. Unanyan, and K. Bergmann, 2002, Eur. Phys. J. D 19, 257.

Gordon, R., L. C. Zhu, and T. Seideman, 1999, Acc. Chem. Res. 32, 1007.

Gottselig, M., D. Luckhaus, M. Quack, J. Stohner, and M. Willeke, 2000, Helv. Chim. Acta 84, 1846.

Grischkowski, D. G., M. M. T. Loy, and P. F. Liao, 1975, Phys. Rev. A 12, 2514.

Guérin, S., and H. R. Jauslin, 1998, Eur. Phys. J. D 2, 99.

Guérin, S., L. P. Yatsenko, T. Halfmann, B. W. Shore, and K. Bergmann, 1998, Phys. Rev. A 58, 4691.

Haché, A., Y. Kostoulas, R. Atanasov, J. L. P. Hughes, J. E.
Sipe, and H. M. van Driel, 1997, Phys. Rev. Lett. 78, 306.

Halfmann, T., and K. Bergmann, 1996, J. Chem. Phys. 104, 7068.

Halfmann, T., L. P. Yatsenko, M. Shapiro, B. W. Shore, and K. Bergmann, 1998, Phys. Rev. A 58, R46.

Haner, M., and W. S. Warren, 1988, Appl. Phys. Lett. 52, 1459.

Hartchcock, M. A., and J. Laane, 1985, J. Phys. Chem. 89, 4231.

Hennrich, M., T. Legero, A. Kuhn, and G. Rempe, 2000, Phys. Rev. Lett. 85, 4872.

Herzberg, G., 1950, Molecular Spectra and Molecular Structure: I. Spectra of Diatomic Molecules, 2nd ed. (Van Nostrand, Princeton).

Hillegas, C. W., J. X. Tull, D. Goswami, D. Strickland, and W. S. Warren, 1994, Opt. Lett. 19, 737.

Hu, J., G. La Rocca, F. Basani, D. Wang, and J. Gao, 2003, Opt. Commun. 216, 157.

Hu, X., and W. Pötz, 1999, Phys. Rev. Lett. 82, 3116.

Huang, G. M., T. J. Tarn, and J. W. Clark, 1983, J. Math. Phys. 24, 2608.

Javanainen, J., and M. Mackie, 1998, Phys. Rev. A 58, R789. Judson, R. S., and H. Rabitz, 1992, Phys. Rev. Lett. 68, 1500.

Kallush, S., and Y. B. Band, 2000, Phys. Rev. A 61, 041401(R).

Kaszlikowski, D., P. Gnacinski, M. Zukowski, W. Miklaszewski, and A. Zeilinger, 2000, Phys. Rev. Lett. 85, 4418.

Kendrick, B., 1997, Phys. Rev. Lett. 79, 2431.

Kis, Z., A. Karpati, B. W. Shore, and N. V. Vitanov, 2004, Phys. Rev. A 70, 053405.

Kis, Z., and S. Stenholm, 2001, Phys. Rev. A 64, 063406.

Kittrell, C., E. Abramson, J. L. Kinsey, S. A. McDonald, D. E. Reisner, R. W. Field, and D. H. Katayama, 1981, J. Chem. Phys. 75, 2056.

Kleiman, V., L. Zhu, X. Li, and R. Gordon, 1995, J. Chem. Phys. 102, 5863.

Knight, P. L., M. A. Lauder, and B. J. Dalton, 1990, Phys. Rep. 190, 1.

Knowles, W. S., 2002, Angew. Chem., Int. Ed. 41, 1997.

Kobrak, M. N., and S. A. Rice, 1998a, Phys. Rev. A 57, 1158.

Kobrak, M. N., and S. A. Rice, 1998b, J. Chem. Phys. 109, 1.

Kobrak, M. N., and S. A. Rice, 1998c, Phys. Rev. A 57, 2885.

Kosloff, R., S. A. Rice, P. Gaspard, S. Tersigni, and D. J. Tannor, 1989, Chem. Phys. 139, 201.

Král, P., 1990a, J. Mod. Opt. 37, 889.

Král, P., 1990b, Phys. Rev. A 42, 4177.

Král, P., 1990c, Czech. J. Phys. 40, 1226.

Král, P., Z. Amitay, and M. Shapiro, 2002, Phys. Rev. Lett. 89, 063002.

Král, P., J. Fiurášek, and M. Shapiro, 2001, Phys. Rev. A 64, 023414.

Král, P., and M. Shapiro, 2001, Phys. Rev. Lett. 87, 183002.

Král, P., and M. Shapiro, 2002, Phys. Rev. A 65, 043413.

Král, P., and M. Shapiro, 2004, Chem. Phys. Lett. 393, 488.

Král, P., and J. E. Sipe, 2000, Phys. Rev. B 61, 5381.

Král, P., I. Thanopulos, and M. Shapiro, 2005, Phys. Rev. A 72, 020303(R).

Král, P., I. Thanopulos, M. Shapiro, and D. Cohen, 2003, Phys. Rev. Lett. 90, 033001.

Král, P., and D. Tománek, 1999, Phys. Rev. Lett. 82, 5373.

Kuhn, A., G. W. Coulson, G. Z. He, S. Schiemann, K. Bergmann, and W. S. Warren, 1992, J. Chem. Phys. 96, 4215.

Kuhn, A., S. Steuerwald, and K. Bergmann, 1998, Eur. Phys. J. D 1, 67.

Kuklinski, J. R., U. Gaubatz, F. T. Hioe, and K. Bergmann, 
1989, Phys. Rev. A 40, 6741.

Kulin, S., B. Saubamea, E. Peik, J. Lawall, M. Leduc, and C. Cohen-Tannoudji, 1997, Phys. Rev. Lett. 78, 4185.

Kurizki, G., M. Shapiro, and P. Brumer, 1989, Phys. Rev. B 39, 3435.

Kurzel, R. B., and J. I. Steinfeld, 1970, J. Chem. Phys. 53, 3293. Kylstra, N. J., E. Paspalakis, and P. L. Knight, 1998, J. Phys. B 31, L719.

Law, C., and J. Eberly, 1998, Opt. Express 2, 368.

Law, C. K., and J. H. Eberly, 1996, Phys. Rev. Lett. 76, 1055.

Li, F.-L., and S.-Y. Zhu, 1999, Phys. Rev. A 59, 2330.

Lu, S.-P., S. Park, Y. Xie, and R. Gordon, 1992, J. Chem. Phys. 96, 6613.

Mackie, M., R. Kowalski, and J. Javanainen, 2000, Phys. Rev. Lett. 84, 3803.

Malinovsky, V. S., and D. J. Tannor, 1997, Phys. Rev. A 56, 4929.

Marquardt, R., M. Quack, and I. Thanopulos, 2000, J. Phys. Chem. A 104, 6129.

Marquardt, R., M. Quack, I. Thanopulos, and D. Luckhaus, 2003, J. Chem. Phys. 118, 643.

Marte, P., P. Zoller, and J. L. Hall, 1991, Phys. Rev. A 44, 4118.

Martin, J., B. W. Shore, and K. Bergmann, 1996, Phys. Rev. A $\mathbf{5 4}, 1556$.

Mayer, R., and H. H. Günthard, 1968, J. Chem. Phys. 49, 1510.

Mayer, R., and H. H. Günthard, 1970, J. Chem. Phys. 50, 353.

McKendry, R., M.-E. Theoclitou, T. Rayment, and C. Abell, 1998, Nature (London) 391, 566.

Mead, C. A., 1992, Rev. Mod. Phys. 64, 51.

Meier, W., G. Ahlers, and H. Zacharias, 1986, J. Chem. Phys. 85, 2599.

Menon, S., and G. S. Agarwal, 1999, Phys. Rev. A 61, 013807. Morris, J. R., and B. W. Shore, 1983, Phys. Rev. A 27, 906.

Moss, D., and C. Parmenter, 1993, J. Chem. Phys. 98, 6897.

Nakajima, T., 1999, Phys. Rev. A 59, 559.

Nielsen, M. A., and I. L. Chuang, 2000, Quantum Computation and Quantum Information (Cambridge University Press, Cambridge, England).

Opatrný, T., and G. Kurizki, 2001, Phys. Rev. Lett. 86, 3180.

Oreg, J., F. T. Hioe, and J. H. Eberly, 1984, Phys. Rev. A 29, 690.

Orr, B. J., J. G. Haub, and R. Haines, 1984, Chem. Phys. Lett. 107, 168.

Park, S., S.-P. Lu, and R. Gordon, 1991, J. Chem. Phys. 94, 8622.

Parkins, A. S., P. Marte, P. Zoller, O. Carnal, and H. J. Kimble, 1995, Phys. Rev. A 51, 1578.

Parkins, A. S., P. Marte, P. Zoller, and H. J. Kimble, 1993, Phys. Rev. Lett. 71, 3095.

Parmenter, C., and B. Stone, 1986, J. Chem. Phys. 84, 4710.

Paspalakis, E., S.-Q. Gong, and P. Knight, 1998, Opt. Commun. 152, 293.

Paspalakis, E., and P. L. Knight, 1998, Phys. Rev. Lett. 81, 293.

Paspalakis, E., M. Protopapas, and P. L. Knight, 1997, Opt. Commun. 142, 34.

Peirce, A. P., M. A. Dahleh, and H. Rabitz, 1988, Phys. Rev. A 37, 4950.

Peřina, J., 1984, Quantum Statistics of Linear and Nonlinear Phenomena (Riedl, Boston).

Quack, M., 1989, Angew. Chem., Int. Ed. Engl. 28, 571.

Quack, M., 1990, Annu. Rev. Phys. Chem. 41, 839.

Raimond, J. M., M. Brune, and S. Haroche, 2001, Rev. Mod. Phys. 73, 565.
Rice, S. A., and M. Zhao, 2000, Optical Control of Molecular Dynamics (Wiley, New York).

Rickes, T., L. P. Yatsenko, S. Steurwald, T. Halfmann, B. W. Shore, N. V. Vitanov, and K. Bergmann, 2001, J. Chem. Phys. 113, 534.

Rodriguez, M., B. M. Garraway, and K.-A. Suominen, 2000, Phys. Rev. A 62, 053413.

Salam, A., and W. J. Meath, 1998, Chem. Phys. 228, 115.

Schleich, W., M. Peringo, and F. L. Kien, 1991, Phys. Rev. A 44, 2172.

Scully, M. O., and M. S. Zubairy, 1997, Quantum Optics (Cambridge University Press, Cambridge, England).

Shapiro, M., and P. Brumer, 1991, J. Chem. Phys. 95, 8658.

Shapiro, M., and P. Brumer, 1999, Adv. At., Mol., Opt. Phys. 42, 287.

Shapiro, M., and P. Brumer, 2003, Principles of the Quantum Control of Molecular Processes (Wiley-Interscience, New York).

Shapiro, M., E. Frishman, and P. Brumer, 2000, Phys. Rev. Lett. 84, 1669.

Shapiro, M., J. W. Hepburn, and P. Brumer, 1988, Chem. Phys. Lett. 149, 451.

Sheehy, B., B. Walker, and L. DiMauro, 1995, Phys. Rev. Lett. 74, 4799.

Shore, B. W., 1990, The Theory of Coherent Atomic Excitation (Wiley, New York), Vol. 1.

Shore, B. W., 1995, Contemp. Phys. 36, 15.

Shore, B. W., K. Bergmann, J. Oreg, and S. Rosenwaks, 1991, Phys. Rev. A 44, 7442.

Solá, I. R., J. Santamarŕia, and V. S. Malinkovsky, 2000, Phys. Rev. A 61, 043413.

Solano, E., G. S. Agarwal, and H. Walther, 2003, Phys. Rev. Lett. 90, 027903.

Süptitz, W., B. C. Duncan, and P. L. Gould, 1997, J. Opt. Soc. Am. B 14, 1001.

Tannor, D. J., R. Kosloff, and S. A. Rice, 1986, J. Chem. Phys. $\mathbf{8 5}, 5805$.

Thanopulos, I., P. Král, and M. Shapiro, 2003, J. Chem. Phys. 119, 5105.

Thanopulos, I., P. Král, and M. Shapiro, 2004, Phys. Rev. Lett. 92, 113003.

Thanopulos, I., and M. Shapiro, 2005, J. Am. Chem. Soc. 127, 14434.

Thanopulos, I., and M. Shapiro, 2006, Phys. Rev. A 74, 031401(R).

Theuer, H., and K. Bergmann, 1998, Eur. Phys. J. D 2, 279.

Theuer, H., R. G. Unanyan, C. Habschied, K. Klein, and K. Bergmann, 1999, Opt. Express 4, 77.

Unanyan, R., M. Fleischhauer, B. W. Shore, and K. Bergmann, 1998, Opt. Commun. 155, 144.

Unanyan, R. G., M. Fleischauer, N. V. Vitanov, and K. Bergmann, 2002, Phys. Rev. A 66, 042101.

Unanyan, R. G., B. W. Shore, and K. Bergmann, 2001, Phys. Rev. A 63, 043405.

Unanyan, R. G., N. V. Vitanov, and K. Bergmann, 2001, Phys. Rev. Lett. 87, 137902.

Unanyan, R., N. V. Vitanov, B. W. Shore, and K. Bergmann, 2000, Phys. Rev. A 61, 043408.

Unanyan, R. G., N. V. Vitanov, and S. Stenholm, 1998, Phys. Rev. A 57, 462.

Unanyan, R., L. P. Yatsenko, K. Bergmann, and B. W. Shore, 1997, Opt. Commun. 139, 48.

Vandersypen, L. M. K., 2001, Nature (London) 414, 883. 
Vardi, A., D. Abrashkevich, E. Frishman, and M. Shapiro, 1997, J. Chem. Phys. 107, 6166.

Vardi, A., M. Shapiro, and K. Bergmann, 1999, Opt. Express 4, 91.

Vitanov, N. V., M. Fleischauer, B. W. Shore, and K. Bergmann, 2001, Adv. At., Mol., Opt. Phys. 46, 55.

Vitanov, N. V., and S. Stenholm, 1997a, Opt. Commun. 135, 394.

Vitanov, N. V., and S. Stenholm, 1997b, Phys. Rev. A 56, 1463.

Vitanov, N. V., and S. Stenholm, 1997c, Phys. Rev. A 56, 741.

Vitanov, N. V., and S. Stenholm, 1999, Phys. Rev. A 60, 3820.

Wang, X., R. Bersohn, K. Takahashi, M. Kawasaki, and H. Kim, 1996, J. Chem. Phys. 105, 2992.

Weiner, A. M., 2000, Rev. Sci. Instrum. 71, 1921.

Weiner, A. M., and J. P. Heritage, 1987, Rev. Phys. Appl. 22, 1619.

Weiner, A. M., D. E. Leiard, G. P. Wiederrecht, and K. A. Nelson, 1990, Science 247, 1317.

Weinstein, Y. S., M. A. Pravia, E. M. Fortunato, S. Lloyd, and D. G. Cory, 2001, Phys. Rev. Lett. 86, 1889.

Winkler, K., G. Thalhammer, M. Theis, H. Ritsch, R. Grimm, and J. H. Denschlag, 2005, Phys. Rev. Lett. 95, 063202.

Yarkony, D. R., 1996, Rev. Mod. Phys. 68, 985.

Yarkony, D. R., 2001, J. Phys. Chem. A 105, 6277.

Yatsenko, L. P., S. Guerin, T. Halfmann, K. Bohmer, B. W. Shore, and K. Bergmann, 1998, Phys. Rev. A 58, 4683.
Yatsenko, L., T. Halfmann, B. W. Shore, and K. Bergmann, 1999, Phys. Rev. A 59, 2926.

Yatsenko, L., B. W. Shore, T. Halfmann, K. Bergmann, and A. Vardi, 1999, Phys. Rev. A 60, R4237.

Yatsenko, L. P., R. G. Unanyan, T. Halfmann, and B. W. Shore, 1997, Opt. Commun. 135, 406.

Ye, C., A. Zibrov, Y. Rostovtsev, A. Matsko, and M. Scully, 2002, J. Mod. Opt. 49, 2485.

Yelin, D., D. Meshulach, and Y. Silberberg, 1997, Opt. Lett. 1793, 22.

Yin, Y.-Y., C. Chen, D. S. Elliott, and A. V. Smith, 1992, Phys. Rev. Lett. 69, 2353.

Yurke, B., and D. Stoler, 1986, Phys. Rev. Lett. 57, 13.

Zepik, H., E. Shavit, M. Tang, T. R. Jensen, K. Kjaer, G. Boldach, L. Leiserowitz, I. Weissbuch, and M. Lahav, 2002, Science 295, 1266.

Zhu, L., V. Kleiman, X. Li, S. Lu, K. Trentelman, and R. Gordon, 1995, Science 270, 77.

Zhu, L., K. Suto, J. Fiss, R. Wada, T. Seideman, and R. Gordon, 1997, Phys. Rev. Lett. 79, 4108.

Zhu, S.-Y., R. Chan, and C. Lee, 1995, Phys. Rev. A 52, 710.

Zhu, S.-Y., H. Chen, and H. Huang, 1997, Phys. Rev. Lett. 79, 205.

Zhu, S.-Y., and M. Scully, 1996, Phys. Rev. Lett. 76, 388.

Zibrov, A., C. Ye, Y. Rostovtsev, A. Matsko, and M. Scully, 2002, Phys. Rev. A 65, 043817. 